\title{
Chemical Modification of Pectin and Polygalacturonic Acid: A Critical Review
}

\author{
Hendryk Würfel, ${ }^{\mathrm{a}}$ Katja Geitel, ${ }^{\mathrm{a}}$ Haisong Qi, ${ }^{\mathrm{b}}$ and Thomas Heinze ${ }^{\mathrm{a}, *}$ \\ Pectin, as a sustainable biopolymer with its two complementary \\ functionalities (carboxyl and hydroxyl moieties) imparted in the $\alpha-1,4-$ \\ galacturonic acid repeating unit, has gained increasing attention in the \\ last few years. The interest in this ubiquitously occurring plant \\ originating polysaccharide (PS) has shifted slowly from applications as \\ a food additive to a broader range of potential applications in medicine, \\ cosmetics, and other industries. Due to the increasing interest in \\ alternatives for petrochemical materials, PSs as biomaterials have \\ gained increasing attention in industrial processes in general. In the \\ last decade, an increasing number of chemical transformations related \\ to pectin have been published, and this is a prerequisite for the design \\ of the structure and hence properties of novel biopolymer-based \\ materials. This work aims to review the chemical modifications of \\ pectin by covalent linkage of the last decade and analyze the materials \\ obtained with these chemical methods critically.
}

Keywords: Pectin; Polygalacturonic acid; Chemical modification; Alkylation; Acylation; Amide; Ester; Hydrazide; Polysaccharide; Hydrogel

Contact information: a: Center of Excellence for Polysaccharide Research, Institute of Organic Chemistry and Macromolecular Chemistry, Friedrich-Schiller-University of Jena, Jena, Germany; b: State Key Laboratory of Pulp and Paper Engineering, South China University of Technology, Guangzhou 510640, China;

* Corresponding author: Tel: +493641 948270, Email: thomas.heinze@uni-jena.de

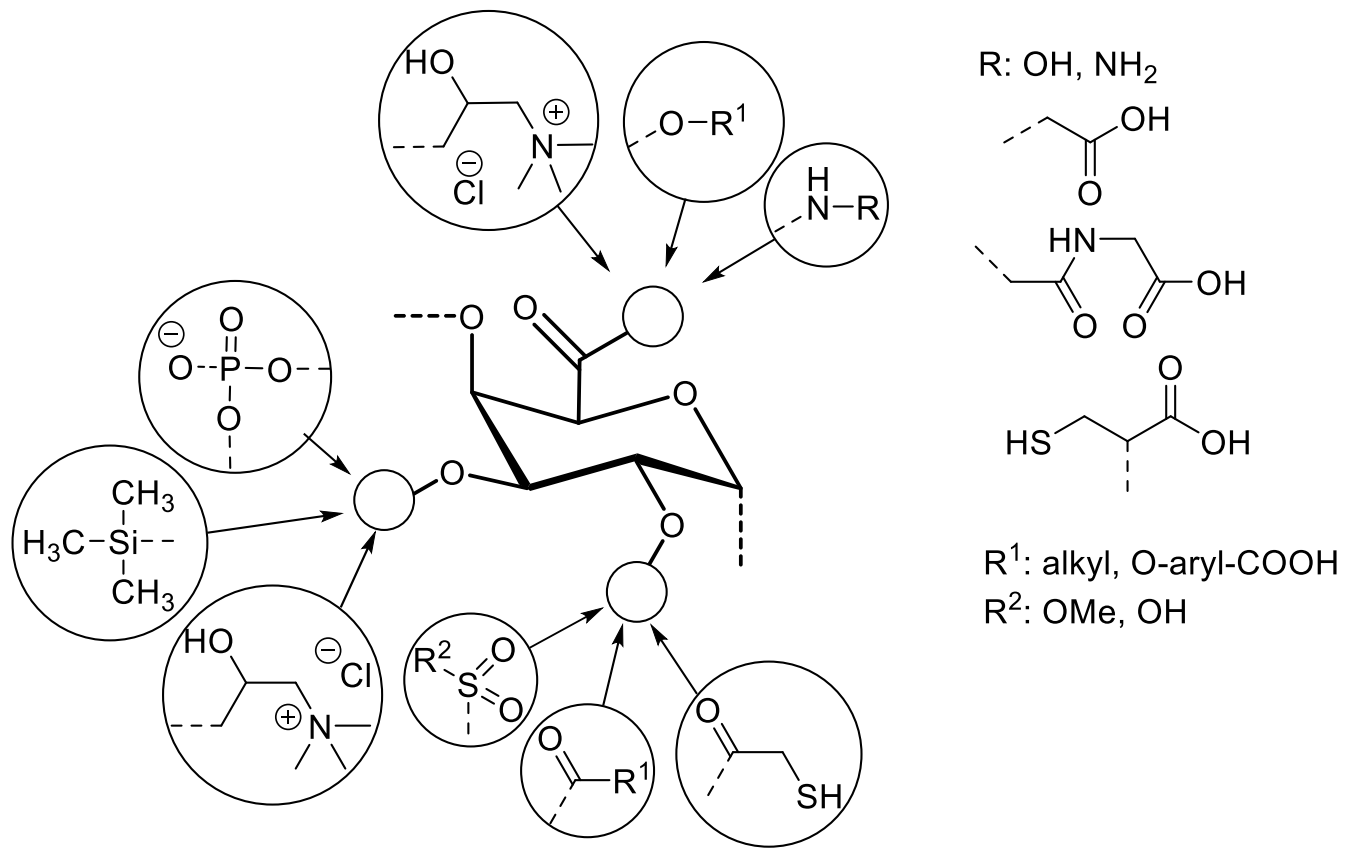

\section{INTRODUCTION}

Pectin, as a plant-based polysaccharide (PS), occurs predominantly in the primary cell wall and the middle lamella and maintains the structural integrity of the cell and provides adhesion between them (Mohen 2008). It was first mentioned in 1790 
by Vauquelin, who isolated the material from tamarind and got its name finally in 1825 (Ropartz and Ralet 2020). Due to its water solubility, the PS can be extracted by a water-based procedure under neutral $\mathrm{pH}$ conditions; however, acidic extraction procedures increase the extraction efficiency dramatically. The structural elucidation of extracted pectin is a laborious and time-consuming procedure even today, due to a large number of neutral sugars imparted (Morris and Binhamad 2020). The material can be considered as a hetero-polysaccharide consisting mainly of $\alpha$-1,4-galacturonic acid chains, which are esterified at the C6-position with a methyl moiety to a varying extent (Fig. 1).

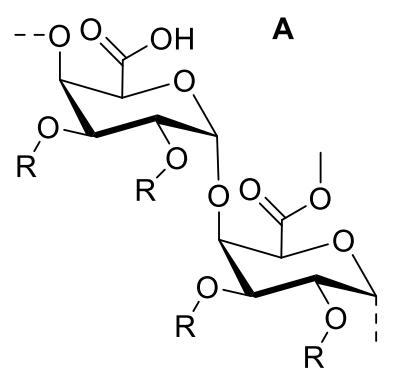

R: H,<smiles>CC(C)=O</smiles>

B

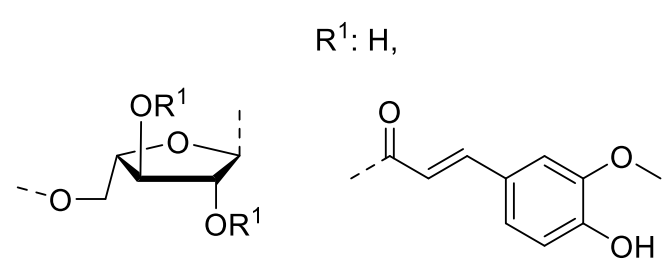

Fig. 1. (A) Main repeating units of pectin consisting of $\alpha-1,4-$ glycosidic linked galacturonic acid. Esterification at C6 with methyl moieties and acetylation at $\mathrm{C} 2$ and $\mathrm{C} 3$ to a varying degree, depending on the polysaccharide source, is observed. (B) Arabinan side chain typically occurring in sugar beet shows acylation with ferulic acid to a varying degree

Additionally, the main chains can be interrupted by $\alpha$-1,2-linked L-rhamnose units, which may possess neutral sugars attached as well. The main features found in naturally occurring pectin are homogalacturonan, rhamnogalacturonan I and II, xylogalacturonan, and apiogalacturonan. These structural features can be summarized with the term multi-block co-biopolymer (Ropartz and Ralet 2020). The predominant homoglacturonan structure can be found as the methyl ester to different degrees. A pectin with less than $50 \%$ of galacturonic acid unit esterified is considered low-methoxy pectin (LMP), whereas with more than 50\% degree of esterification it is high-methoxy pectin (HMP) (Seymour and Knox 2002). The degree of esterification at the C6 position has a large influence on the ability to form gels. HMP can form gels at low $\mathrm{pH}$ values in the presence of high concentration of neutral sugars. This property is utilized for the preparation of fruit jellies and jams. LMP forms gels with multivalent metal ions in aqueous solution. Mostly calcium ions have been employed for this purpose (Williams 2020).
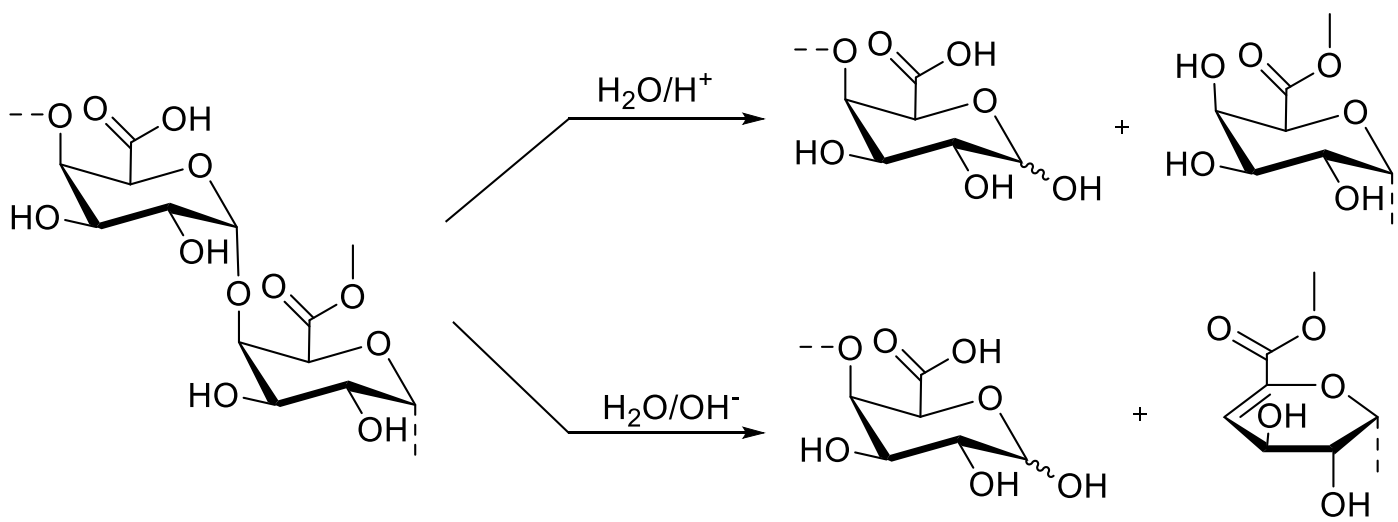

Scheme 1. Two mechanisms for pectin chain degradation occurring at low (top) and high (bottom) $\mathrm{pH}$ values. At low $\mathrm{pH}$ values the glycosidic bond is cleaved, at high $\mathrm{pH}$ values a $\beta$ elimination occurs preferably at repeating units bearing a methyl ester moiety. 
Additional groups found in naturally occurring pectin are ferulic and acetic acid. They have a negative effect on the water solubility of the PS and the ability to form gels with metal ions (Kouwijzer et al. 1996). A chemical modification of pectin frequently occurring as a side reaction is the ester cleavage in basic or acidic aqueous systems. In these procedures also the ferulic- and acetic acid moieties bound to the pectin are cleaved, leaving a LMP. These procedures have to be considered critically, due to the $\mathrm{pH}$ sensitivity of the pectin chain, which leads to depolymerization (Albersheim et al. 1960). In the case of acidic treatment, the glycosidic bond can be cleaved (Scheme 1, top). In basic medium, a $\beta$-elimination leads to fission of polymer chains as well (Scheme 1, bottom).

Although a review dealing with modification of pectin was published in 2015, which describes, besides chemical, also physical transformations (degradation) of the PS (Chen et al. 2015), it was reasonable to review the state of the art in this field due to the increasing popularity of PS as feedstock for sustainable materials. The present review is focused on chemical transformations of pectin and the products obtained, considering preferably the last 10 years of pectin research including correct chemical terminology. A common term used in PS chemistry is the degree of substitution (DS). The DS describes the (average) number of substituent groups attached per repeating unit of the PS. For cellulose, the value ranges from $\mathrm{DS}=0$ to 3 , whereas for pectin from $\mathrm{DS}=0$ to 2 (EPST 2011). The amount of chemical modification at the carboxylic acid function of pectin is commonly expressed in percent conversion or degree of formation, not to be confused with the DS value. No degradation reactions have been specifically described. However, it can be assumed that -at least partially- pectin depolymerization occurs in the course of most of the reactions mentioned here. The review is divided into chemical reactions considering the carboxylic acid function at the $\mathrm{C} 6$ position and reactions considering the hydroxyl groups of pectin. In the last part, reactions not fitting in these categories are presented. Additionally, some trends concerning pectin and its applications are presented as well.

\section{Formation of Esters at $\mathbf{C 6}$}

The ester cleavage of the galacturonic acid unit of pectin is a side reaction occurring in extraction of pectin (Krall and McFeeters 1998; Diaz et al. 2007). The content and the type of ester makes it possible to modify the water solubility, viscosity, and gelling behavior of pectin (Flutto 2003). Besides the introduction of a hydrophobic group, which lowers the water solubility, additional functional groups can be introduced as well. Recent methods involve working with alkylation agents and employing the carboxylate form of pectin to obtain ester functions. The carboxylate reacts as a nucleophile with the alkyl halide. Zheng et al. (2013) employed a heterogeneous approach, comparable with the conditions of carboxymethylation of cellulose (Heinze et al. 2018). A highly esterified pectin was dispersed in 2-propanol and an excess of aqueous $\mathrm{NaOH}$ added (Scheme 2).
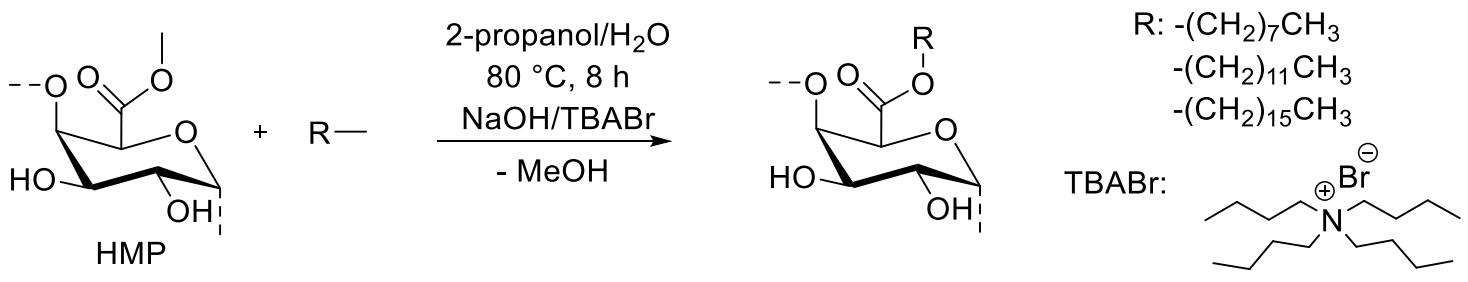

Scheme 2. Esterification of pectin in basic medium with long chain alkyl bromides at elevated temperatures in the presence of tetrabutylammonium (TBA) bromide as catalyst 
To the mixture, the catalyst tetrabutylammonium (TBA) bromide and long chain alkyl bromides were added. The reaction proceeded at $80{ }^{\circ} \mathrm{C}$, yielding low amounts of carboxylic acid ester only. The content of carboxylic acid moieties esterified ranged from $4 \%$ up to $14 \%$. The water solubility decreases with increasing amount of ester formed, due to the introduction of these hydrophobic alky groups. In this procedure, TBA bromide was found to be a good phase transfer catalyst, which leads to the formation of hydrophobic pectin derivatives. Investigations showed that in the absence of the catalyst or a change of the counter ion of the ammonium salt to hydroxide, the reaction did not produce the desired hydrophobic pectin.

To perform a homogeneous alkylation of pectin in dimethyl sulfoxide (DMSO), the solubility of pectin had to be adjusted. Usually salts of pectin (alkali ions) are insoluble in DMSO. However, the TBA salt of pectin can be dissolved in this dipolar aprotic solvent (Renard and Jarvis 1999). By neutralizing pectin in aqueous solution with TBA hydroxide and subsequent lyophilization, a DMSO-soluble pectin salt can be obtained. The salt dissolved in DMSO was reacted with long chain alkyl bromides with $\mathrm{C}_{6}, \mathrm{C}_{12}$, and $\mathrm{C}_{18}$ alkyl chains (Liang et al. 2015). The alkyl ester formation with the alkyl bromides $\left(\mathrm{C}_{6}\right)$ reached $0.7 \%, 2.4 \%$, and $3.6 \%$, respectively. To compare pectin with longer alkyl chain ester moieties as well, derivatives with $\mathrm{C}_{12}$ and $\mathrm{C}_{18}$ have been prepared from their corresponding alkyl bromides as well. The esterified compounds possessed a degrees of esterification of $0.7 \%$. Interestingly the molecular masses were comparable after synthesis, as has been shown by high performance size exclusion chromatography measurements. The intrinsic viscosity $[\eta]$ was found to decrease with increasing alkyl chain length. On the contrary, their apparent viscosities increased with increasing length of the alkyl chain attached. The emulsion stabilizing effects of the esters obtained have also been probed and were shown to be correlated with the alkyl chain length of the ester moiety. The longer the alkyl chain attached to the pectin, the smaller the emulsified droplets turned out to be. Additionally, an increase in alkyl chain length of the pectin ester increased the stability of the emulsions formed, which was probed for a period of 7 days.

An enzymatic esterification of the carboxylic acid function of pectin could be achieved when employing laccase as an enzyme catalyst (Karaki et al. 2017). The reaction was performed in phosphate buffer at $30^{\circ} \mathrm{C}$, leading to an esterification of the hydroxyl group of ferulic acid with the $\mathrm{C} 6$ carboxylic function of pectin with a conversion of $6 \%$. The low degree of esterification was difficult to detect with infrared (IR) and ${ }^{13} \mathrm{C}$ nuclear magnetic resonance (NMR) spectroscopy. However, UV/Vis spectroscopy could show differences between the native and transformed pectin, due to the increase of aromatic moieties in the latter (Scheme 3). The study shows that the preparation of pectin derivatives with potentially antioxidant properties is possible, e.g., for the use in the food industry.
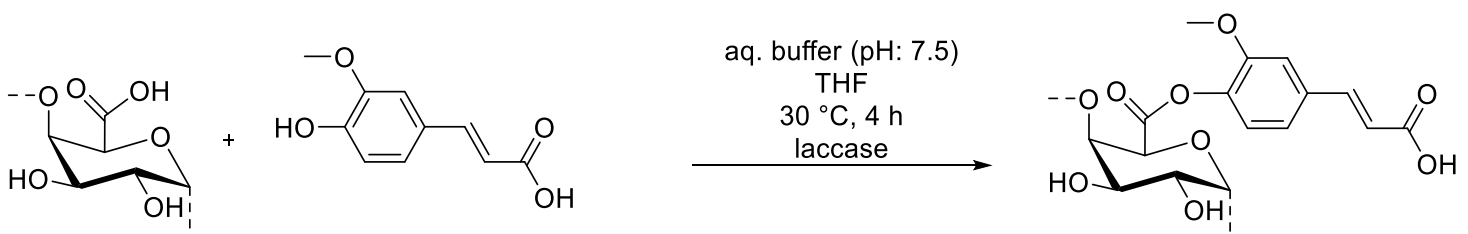

Scheme 3. Enzymatic esterification of pectin with ferulic acid in an aqueous buffer/tetrahydrofuran (THF) mixture at $30^{\circ} \mathrm{C}$

Along these lines, other phenolic acid derivatives closely related to ferulic acid have been enzymatically esterified with pectin as well (Zhang et al. 2020a). Gallic acid was bound via its 4-OH group with the $\mathrm{C} 6$ carboxylate of pectin employing a lipase 
(Novozyme 435, from Aspergillus niger) in a water/tetrahydrofuran mixture at $50{ }^{\circ} \mathrm{C}$. Subsequently p/m/o-hydroxybenzoic acid and 3,4-dihydroxy benzoic acid have been reacted in a similar manner (Scheme 4, Zhang et al. 2020b). The materials obtained have been tested for their emulsifying properties as well as their antioxidant and antibacterial activities.

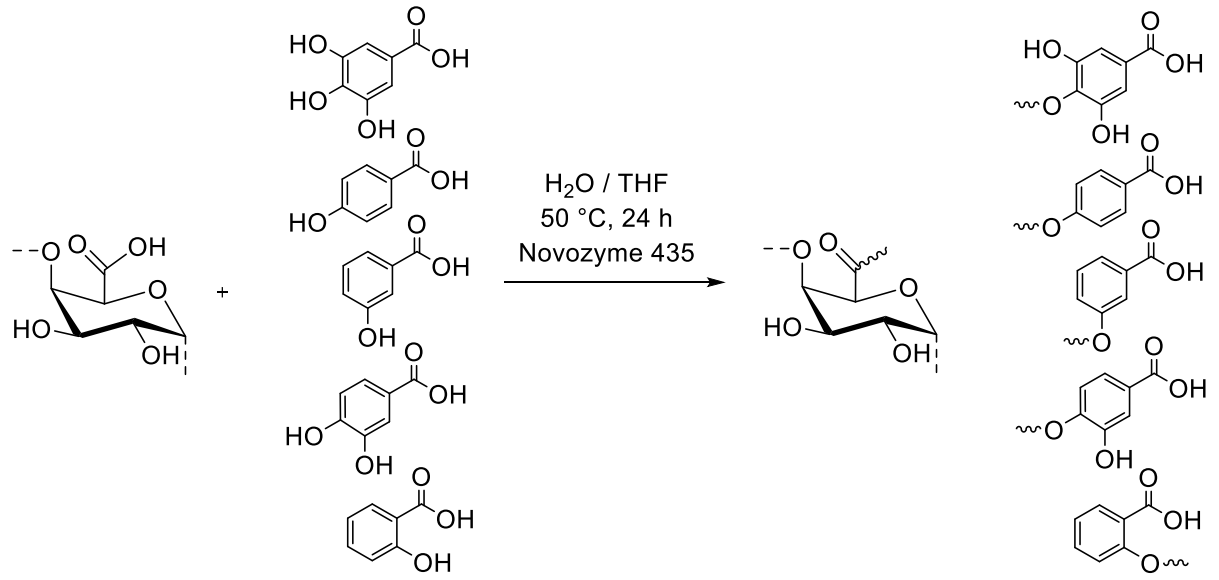

Scheme 4. Enzymatic esterification of pectin with different hydroxybenzoic acid derivatives in a water/tetrahydrofuran (THF) mixture at $50^{\circ} \mathrm{C}$
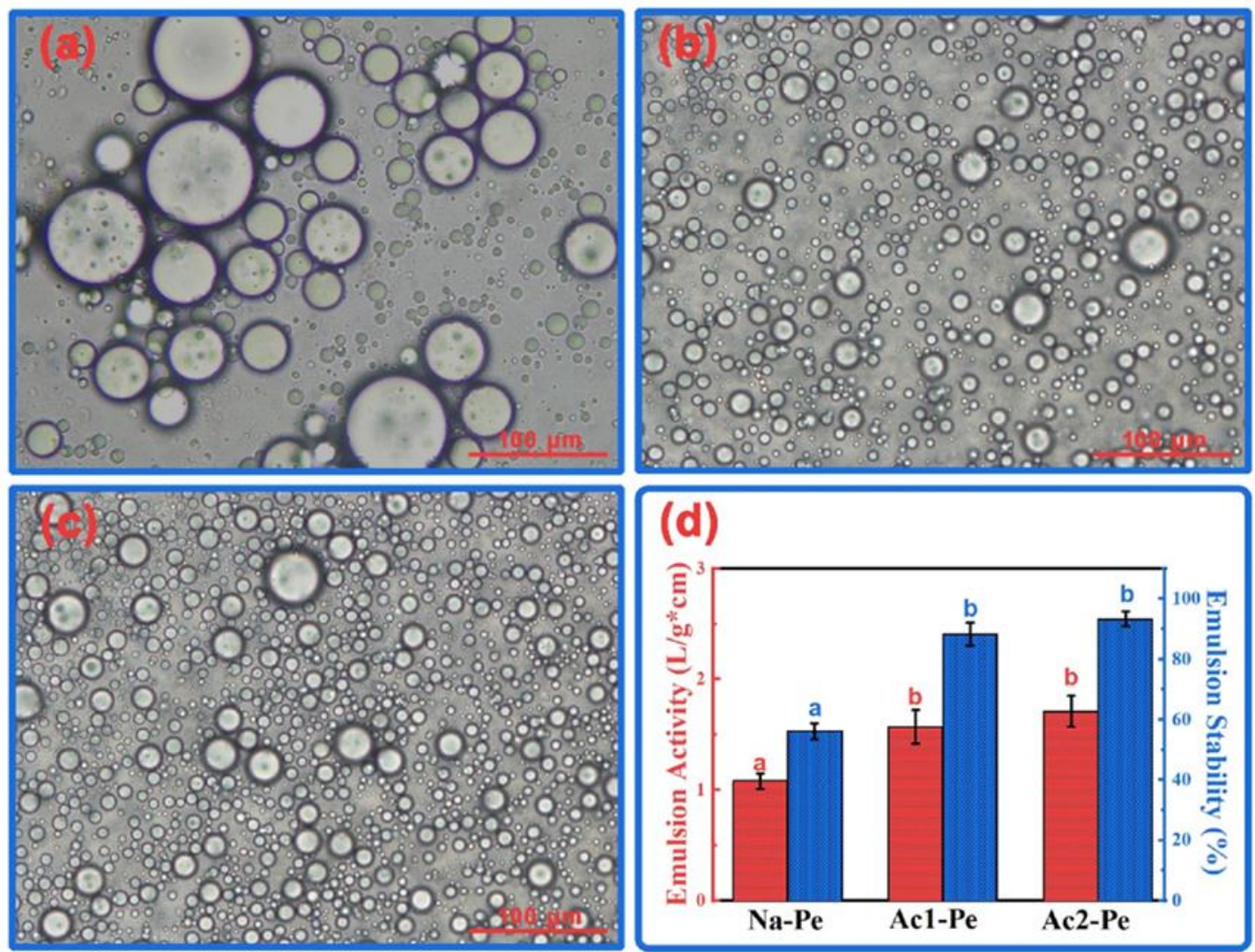

Fig. 2. Emulsifying properties of the starting pectin (a) and the pectin benzoic acid ester derivatives (b, c). Reprinted from International Journal of Biological Macromolecules, 165, Zhang, G., Zheng, C., Huang, B. and Fei, P., "Preparation of acylated pectin with gallic acid through enzymatic method and their emulsifying properties, antioxidation activities and antibacterial activities", p. 202, Copyright (2020) with permission from Elsevier 
The materials esterified showed an increase in emulsifying properties, e.g., decreased droplet size and prolonged droplet stability in contrast to the staring pectin, due to the amphiphilic nature of the hydrophobic aromatic carboxylic acid moiety introduced (Fig. 2). Moreover, they exhibited a clear inhibition zone in contrast to the native pectin when probing their antibacterial properties against E. coli and S. aureus. Interestingly in these investigations, no cross-linking or esterification of the benzoic acid derivatives with the hydroxyl groups of pectin was observed.

An esterification of the carboxylic acid group of pectin was also achieved by employing a photoactive diazo-resin (Plewa et al. 2011). The material, synthesized in a condensation reaction of diphenylamine-4-diazonium salt and paraformaldehyde, can generate phenyl cations under UV light irradiation. These cations are prone to nucleophilic attack from a carboxylate group, forming phenyl esters. By this photochemical reaction, pectin and the diazo resin could be cross-linked permanently. The reaction was monitored and quantified directly employing UV/Vis spectroscopy. The two polymers have been deposited in multiple layers on quartz plates and onto silica gel particles as well for the transformation and were subsequently cross-linked with UV light at $350 \mathrm{~nm}$ (Scheme 5). The resulting materials were shown to be promising scaffolds for growing cell cultures especially for bone tissue engineering.
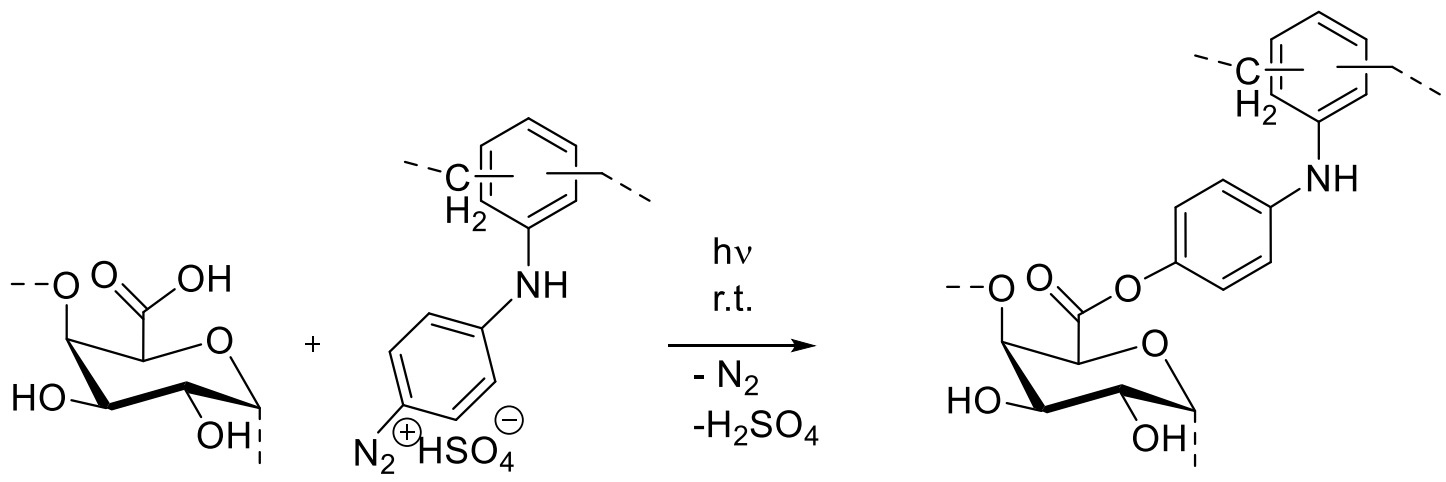

Scheme 5. Formation of pectin ester followed by cross-linking of pectin and a diazo resin triggered by irradiation with UV light at $350 \mathrm{~nm}$

\section{Heteroatom Transformation at the Carboxylic Acid Group}

Formation of amide

Pectin amide is a well-known thickening agent that is safe for use in the food industry (Mortensen et al. 2017). It can be produced simply by reacting native pectin with ammonia, thus cleaving the methyl ester moieties releasing methanol (EinhornStoll et al. 2001). Structurally more diverse amides of pectin have been synthesized in the last few years.

An increased functionality of pectin derivatives can be achieved by reacting HMP with amino acids. The amide structures formed are stable chemical bonds, and the other functionalities of the amino acid moiety are still accessible. Kurita et al. (2012) reported on this transformation; HM citrus pectin and glycine, or glycine methyl ester, and glycylglycine were reacted in aqueous solution at $\mathrm{pH}$ value of 5.4 (Scheme 6 ). The amide formation occurred to an extent of $1 \%$ to $5 \%$ only, not changing the solubility of the biopolymer derivatives in water to a large extent. It is reasonable to assume that the water employed caused the low degree of amide formation due to hydrolysis of the pectin methyl ester in the acidic medium. 


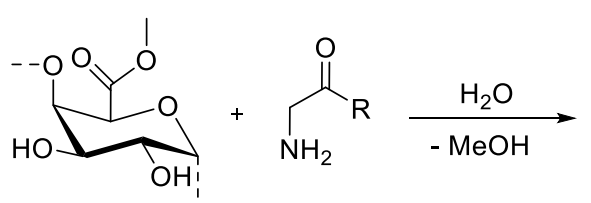<smiles>[R]C(=O)CNC(=O)C1OC(O)C(O)C(O)C1O</smiles>

R: $\mathrm{OH}$ (glycin)

$\mathrm{R}$ : OMe (glycine methylester)

R: $\mathrm{NHCH}_{2} \mathrm{COOH}$ (glycylglycine)

Scheme 6. Formation of pectin amides from high-methoxy pectin and glycine derivatives in water

The amide formation was also studied with cysteine and a LMP, thus incorporating thiol functional groups in the pectin structure (Chen et al. 2018; Eliyahu et al. 2021). The carboxylic acid function of pectin was activated in water using 1-ethyl3-(3-dimethylaminopropyl)carbodiimide (EDC). The activated PS was reacted with Lcysteine monohydrate hydrochloride at a $\mathrm{pH}$ value of 4.75 to obtain the corresponding pectin amide (Scheme 7). The product fabricated was mixed with a chitosan acrylate, and the gel formation was studied in detail. Two cross-linking mechanisms could be identified. An electrostatic cross-linking was achieved, combining the carboxylate groups of pectin with the ammonium groups of the chitosan. A Michael addition of the thiol groups (Michael donor) to the electron-deficient double bond of the acrylamide moieties (Michael acceptor) resulted in a slow increasing permanent cross-linking of the two polymers mixed.

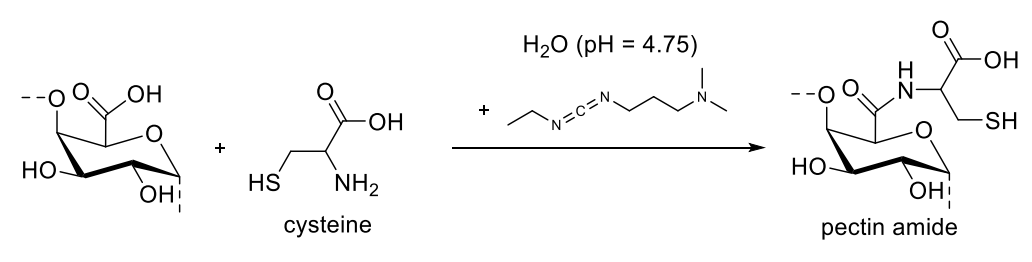

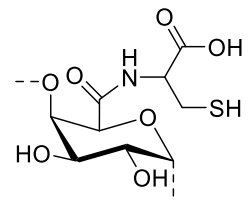

pectin amide

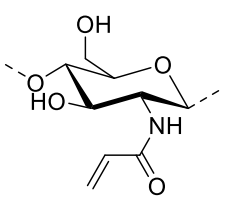

chitosan acrylate

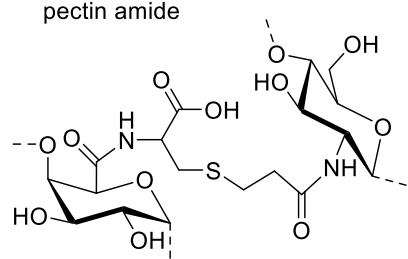

Scheme 7. The carboxylic acid function of pectin was activated in water with 1-ethyl-3-(3dimethylaminopropyl)carbodiimide and reacted with cysteine to form a pectin amide (top). A Michael addition between the thiol group of the pectin derivate and a chitosan acrylate resulting in a cross-linked gel (bottom)
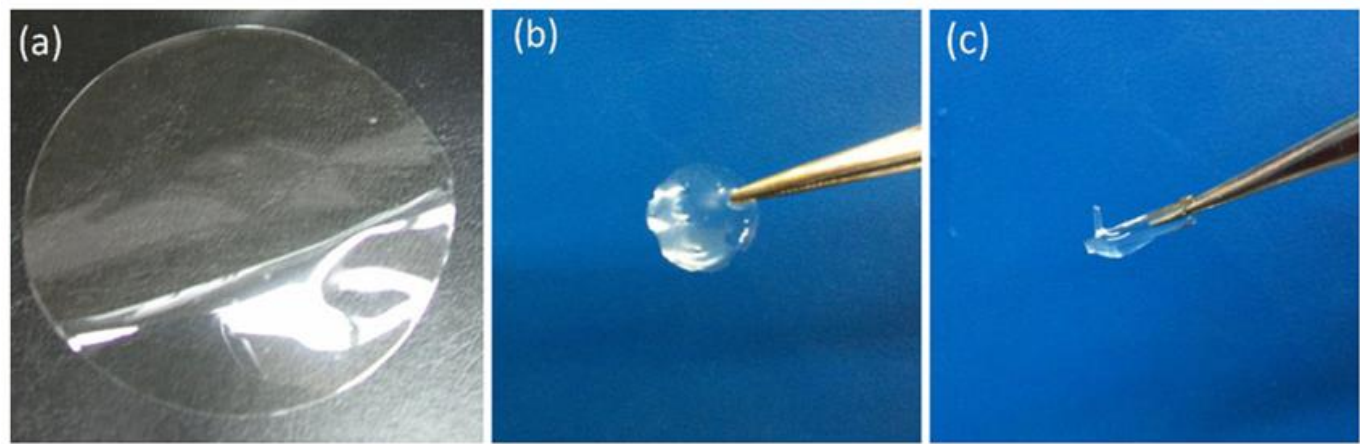

Fig. 3. Anti-adhesive films based on disulfide cross-linked cysteine moieties bearing polygalacturonic acid amide. The material is shown in the dry state $(A)$, immersed in phosphate buffered saline solution (B), and as a comparison a hyaluronate/carboxymethylcellulose gel (C). Reprinted from J. Mater. Sci.: Materials in Medicine, 24(6), Peng, H.-H., Chen, Y.-M., Lee, C.-I. and Lee, M.-W., "Synthesis of a disulfide cross-linked polygalacturonic acid hydrogel for biomedical applications", p. 1378, Copyright (2013), with permission from Springer Nature. 
The influence of temperature and $\mathrm{pH}$ value on the curing time and gel properties was investigated, showing that the increase of $\mathrm{pH}$ value gave stiffer gel structures and $\mathrm{pH}$ values below the $\mathrm{pKa}$ of chitosan led to materials with greatly enhanced adhesiveness. The gels are potential candidates for mucosa-mimetic materials (Fig. 3).

An amide-linked polygalacturonic acid-cysteine material was prepared with a degree of modification of 16\%, which forms hydrogels via disulfide cross-linking (Peng et al. 2013). The hydrogel obtained could be employed as a carrier for rosmarinic acid and used to prevent postsurgical adhesion and inflammatory reactions. It was shown to significantly reduce adhesion incidents (over 90\%); on the contrary hyaluronate/ carboxymethylcellulose leads to a reduction of $42 \%$ only.

To protect the thiol group against oxidation at $\mathrm{pH}$ values above 5 , a cysteinenicotinic acid disulfide was synthesized (Hintzen et al. 2013). This product was allowed to react with pectin after activation of the C6 carboxylic group with EDC in water. In addition to the EDC activation of the carboxylic acid, $N$-hydroxysuccinimide was added, probably to form an activated ester in situ right before the amide formation (Scheme 8). The resulting materials were shown to be non-cytotoxic on Caco-2 cells within $24 \mathrm{~h}$. The S-protection improved the stability as well as the adhesive and cohesive properties of the pectin derivative compared to the unprotected pectin derivative. Comparable products were investigated for a drug release system, employing lidocaine as a model drug (Hauptstein et al. 2013). The gel formation of the starting pectin, pectin cysteine amide, and thiol protected pectin-cysteine amide were compared, and the swelling behavior and drug release of these gels in a saline buffer solution was probed for possible application in the buccal tissue. The thiol protected pectin-cysteine amide showed the best drug release behavior in comparison to the other two biopolymer derivatives investigated.

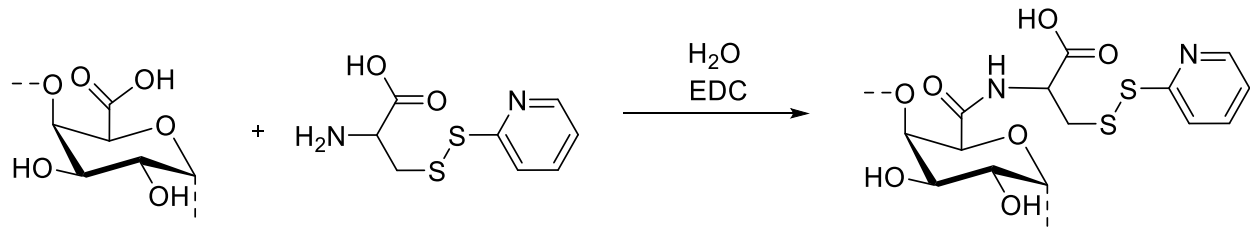

Scheme 8. Pectin amide formation with S-protected cysteine in water aided by activation of the carboxylic acid function by 1-ethyl-3-(3-dimethylaminopropyl)carbodiimide (EDC)

In a more recent study, amino acids (glutamic acid, glycine, cysteine, lysine, and arginine) were allowed to react with HMP without any catalyst in water at $40{ }^{\circ} \mathrm{C}$ (Chen et al. 2020). From the amino acids tested, only glycine, lysine, and arginine could form amide bonds with the pectin. The degree of amide formation was generally low, not exceeding $6.5 \%$. The pectin derivatives were probed using elemental analysis, Fourier transform infrared (FTIR), and ${ }^{1} \mathrm{H}$ NMR spectroscopy. The surface topographies of the samples prepared showed appreciable differences as revealed by environmental scanning electron microscopy (SEM) (Fig. 4). The amino acid-pectin amide derivatives possess no cytotoxic effects, which would be expected considering pectin and amino acids are already non-cytotoxic.

The formation of a carboxylic acid amide with taurine led to a product that exhibits good surfactant properties (Aris et al. 2017). Taurine can be linked to polygalacturonic acid (PGA) via activation with EDC or without activation by treating the reaction mixture in a microwave oven (Scheme 9). The amount of carboxylic acid amide formed corresponding to the available carboxylic acid functions was $10 \%$. The product exhibited surface activity comparable to Triton X-100 and, additionally, it is non-toxic against human dermal fibroblast and human leukemic cell lines. 


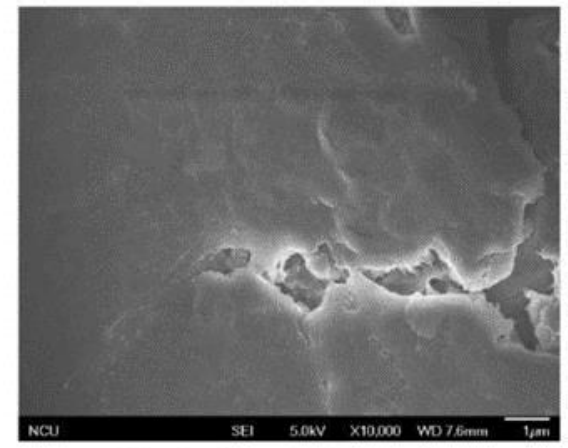

Original Pectin

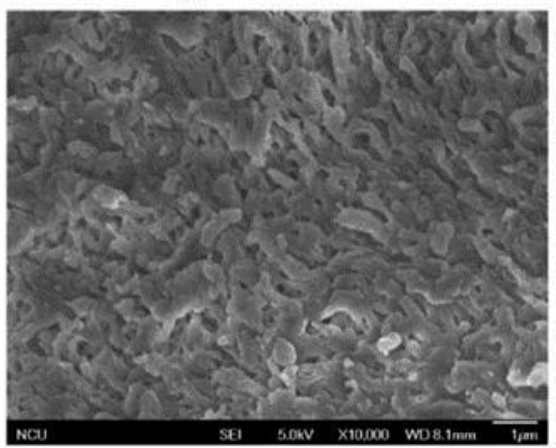

P-Lys

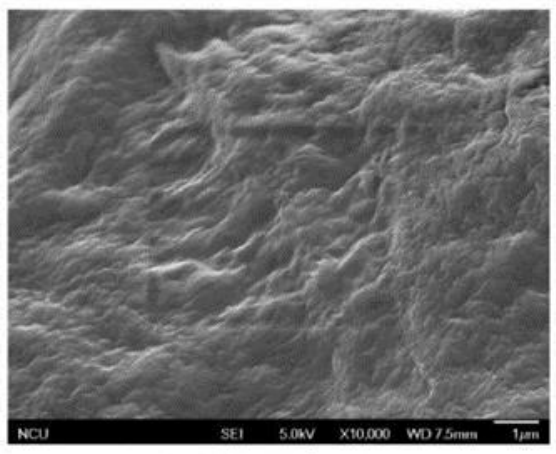

P-Gly

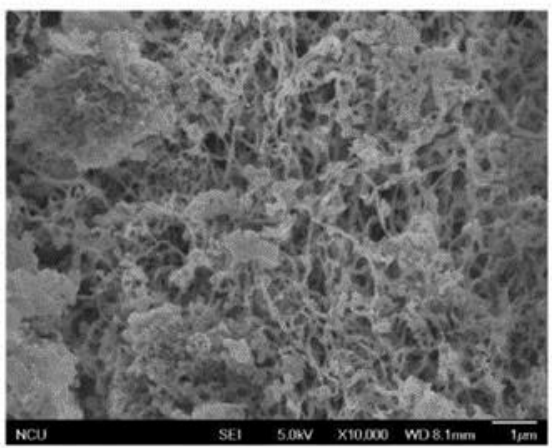

P-Arg

Fig. 4. Environmental scanning electron microscopy images of the starting pectin, the pectin amide of glycine (P-Gly), the lysine (P-Lys) and the arginine derivative (P-Arg) of pectin after freeze-drying. Reprinted from Journal of Food Chemistry, 309, Chen, J., Niu, X., Dai, T., Hua, H., Feng, S., Liu, C., "Amino acid-amidated pectin: Preparation and characterization,” p. 5, Copyright (2020), with permission from Elsevier<smiles>COC(=O)CC(O)C(O)C(O)C(O)C(=O)O</smiles><smiles>NCCS(=O)(=O)O</smiles><smiles>O=COCCOCCO</smiles><smiles>COC1CC(=O)NCCS(=O)(=O)OC1C(O)O</smiles>

Scheme 9. Formation of pectin amide of taurine in water, 1-ethyl-3-(3-dimethylamino-propyl) carbodiimide (EDC)

The synthesis route employing LMP with activation in aqueous medium via EDC was also followed by Tang et al. (2010). They used this method to form pectin amide with adriamycin, a drug employed in treating different types of solid malignant tumors. After dissolving all components, the reaction proceeded at $50{ }^{\circ} \mathrm{C}$ for $8 \mathrm{~h}$. The dialyzed and lyophilized product was transformed into nanoparticles (NP) that showed a narrow distribution with the main peak at $152 \mathrm{~nm}$ and a Z-average diameter of 126 $\mathrm{nm}$, which is a good value for the necessary renal clearance after NP administration. The drug release at $37{ }^{\circ} \mathrm{C}$ was increased by decreasing the $\mathrm{pH}$-value from 7.4 to 5.0. The addition of lysosomes to the pectin derivative solution also had a positive drug releasing effect. Unfortunately, the study revealed that at concentrations equivalent to 0.125 to $1.000 \mu \mathrm{g}$ of pectin derivative/mL, it did not inhibit the growth of either A594 or B16 cells to the same extent as the free drug or a mixture of the free drug with pectin. 
However, an anticancer effect on C57BL/6 mice was superior for the drug loaded pectin derivative in contrast to the drug alone or a mixture of the drug and pure pectin.

Using a water/dioxane mixture and EDC as activating agent, Perera et al. (2010) were able to form amide bonds between the carboxylic acid function of pectin and 4-aminothiophenol. The idea was to employ the thiophenol moiety as a reversible cross-linker in an oxidation/reduction procedure. Two derivatives were synthesized with a degree of amidation of approximately $3 \%$ and $10 \%$. The viscosity of the sample dissolved was measured before and after the addition of hydrogen peroxide. It was found that a large increase in viscosity appeared. For the pectin derivatives containing $3 \%$ and $10 \%$ of amide groups, a 29 -fold and 500-fold increase in dynamic viscosity was found, after $60 \mathrm{~min}$ of peroxide treatment. The materials synthesized showed increased water uptake after cross-linking. Additionally, no increase in toxicity compared to the starting PS was observed. Due to the increased PS stability after crosslinking, these pectin derivatives might be of interest for colon drug delivery systems.

\section{Formation of hydroxamic acid}

The reaction of hydroxylamine and HMP conducted in water at room temperature (RT) for $4,18,24$, or $48 \mathrm{~h}$ resulted in the formation of a hydroxamic acid (Bae et al. 2011). The content of hydroxamic acid ranged from $4.7 \%$ to $10.4 \%$ conversion and increased with increasing reaction time (Scheme 10). In contrast to the highly esterified starting material, the hydroxamic acid derivatives produced showed an increased water solubility, which can be attributed to the decrease in hydrophobic methyl ester moieties. The inhibitory activity of pectin hydroxamic acid derivatives against semicarbazide-sensitive amine oxidase and angiotensin-converting enzyme was shown earlier (Hou et al. 2003).
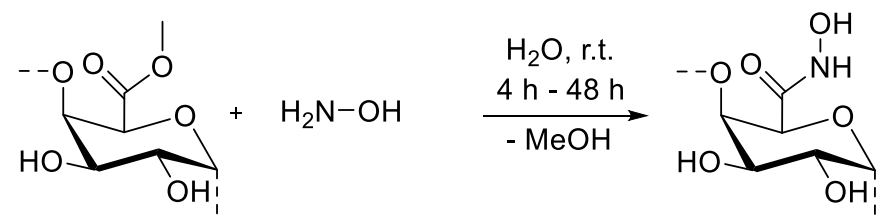

Scheme 10. Formation of a hydroxamic acid derivative of pectin by reaction of a highly methyl esterified pectin in water with hydroxylamine

\section{Formation of hydrazide}

A homogeneous synthesis of pectin hydrazide was presented by Guo et al. (2014). The transformation of HMP was studied in aqueous solution with a large excess (60 eq.) of hydrazide hydrate at $80{ }^{\circ} \mathrm{C}$ for $12 \mathrm{~h}$ (Scheme 11). The hydrazine hydrate reacted with the methyl ester groups of pectin, forming the corresponding hydrazide in only moderate yields. The product showed an increased nitrogen content and was able to bind mercury ions from an aqueous solution more efficiently than the starting HMP.

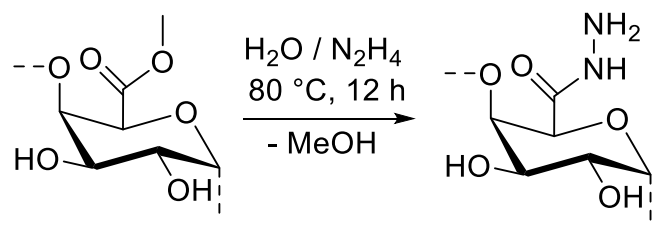

Scheme 11. Homogeneous reaction of high-methoxy pectin with hydrazine hydrate in water at elevated temperatures

A heterogeneous synthesis approach for pectin hydrazide could be realized as well and was shown to be more efficient and even useful for industrial application 
(Würfel et al. 2021). The reaction of LMP or PGA proceeds in a slurry of 2-propanol between $\mathrm{RT}$ and $50{ }^{\circ} \mathrm{C}$, applying 4 eq. to 10 eq. of hydrazide hydrate. The reaction is complete after $4 \mathrm{~h}$, leading to a hydrazide content of up to $98 \%$. Interestingly, no methyl ester groups are present or needed for this transformation. The carboxylic acid groups or their ammonium salts react readily with hydrazine hydrate while releasing water or ammonia by forming the correspondent hydrazide (Scheme 12). The pectin hydrazide thus obtained had a good metal chelating ability and can form stable gels with multivalent metal ions. Furthermore, an ex ovo hen's egg test demonstrated that the pectin hydrazide derivatives are non-toxic.
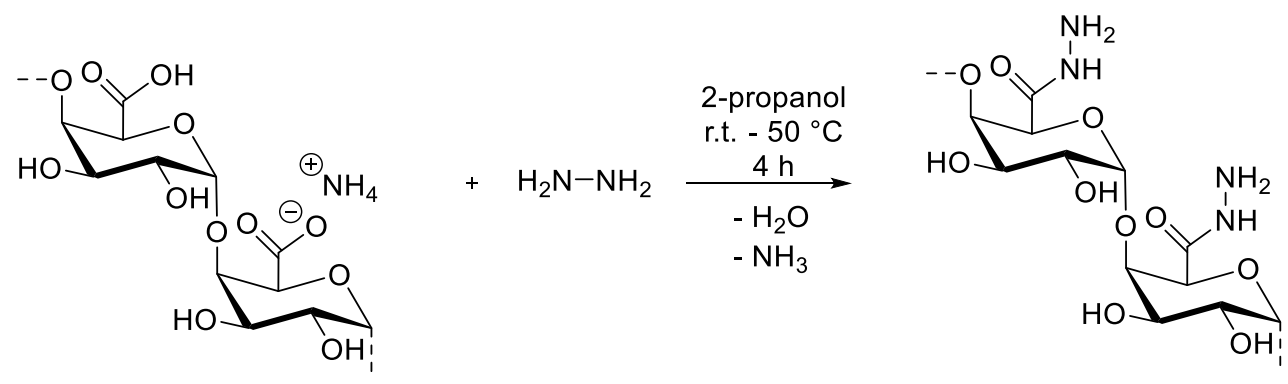

Scheme 12. Formation of polygalacturonic acid hydrazide from ammonium polygalacturonate heterogeneously in 2-propanol

\section{Acylation Reaction at the Hydroxyl Group}

Native pectin incorporates varying amounts of acyl groups, originating from acetic- and ferulic acid groups. The acetylation of pectin was investigated and was shown to have a strong influence on the water solubility and the ability to form gels with multivalent metal ions (Wang et al. 2019). Different strategies have been employed to acylate pectin with a variety of carboxylic acid derivatives. A solvent-free approach was published for esterifying the hydroxyl groups of pectin with fatty acid anhydrides catalyzed with base at $160{ }^{\circ} \mathrm{C}$ (Monfregola et al. 2011). The compounds were ground in an agate mortar and heated in an oil bath for 15 to 20 min (Scheme 13). The resulting pectin esters have been isolated by extracting the reaction product with chloroform. This method of purification contradicts the claim of an environmentally friendly method of esterification, as stated. The reaction yields pectin esters of low DS of 0.27 for hydroxyl oleic acid anhydride and DS $=0.07$ for linoleic acid anhydride.
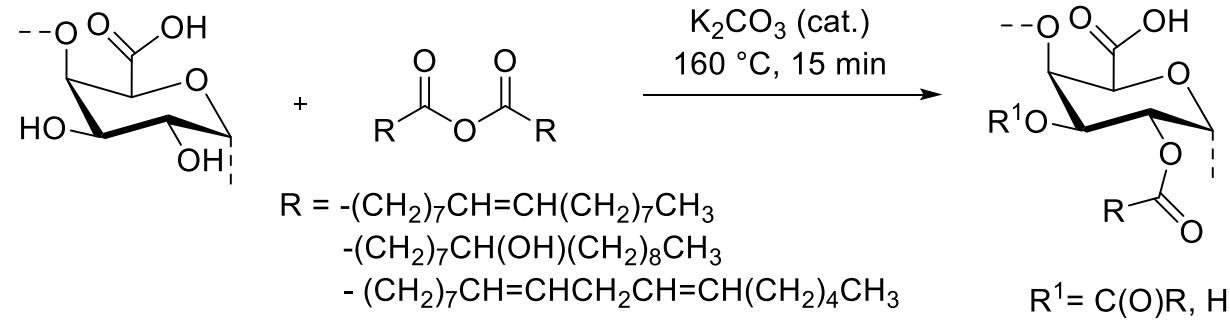

Scheme 13. Solvent-free and base-catalyzed acylation of pectin with long chain acid anhydrides at $160^{\circ} \mathrm{C}$ for $15 \mathrm{~min}$

Later, a similar microwave assisted approach was published (Calce et al. 2012). The mixture of pectin, fatty acid anhydride, and $\mathrm{K}_{2} \mathrm{CO}_{3}$ was ground and treated subsequently in the microwave with $900 \mathrm{~W}$ for $3 \mathrm{~min}$ to $6 \mathrm{~min}$. The products were still water soluble and possessed an intense IR absorption belonging to the $\mathrm{C}-\mathrm{H}$ stretching vibrations of the fatty acid moieties introduced. The anhydrides of linoleic acid, oleic acid, and palmitic acid were employed. As interesting as this synthesis protocol seems 
to be, the reactions was performed in amounts of $30 \mathrm{mg}$ only, showing problems arising from solvent-free procedures such as homogeneous mixing of the compounds employed and efficient absorption of the microwave irradiation by materials with low dielectric properties.

Pectin derivatives incorporating linoleic-, oleic-, and palmitic acid esters were found to be active against different strains of bacteria (Calce et al. 2014). Pectin oleate and -linoleate could inhibit the growth of $S$. aureus and E. coli by $50 \%$ to $70 \%$. Furthermore, the pectin esters could be coated on polyethylene films that possess good oxygen barrier properties, which could be interesting in the field of food application and packaging.

The reaction of HMP with diacylhalides (glutaryl chloride and sebacoyl chloride) was described (Seslija et al. 2018). The homogeneous transformation with the acylation reagent proceeds in DMSO and pyridine applying a ratio of reagent/pectin of 1:3 and 1:15 (Scheme 14). The conversion rate was low. Otherwise the resulting product would have been insoluble in water, due to cross-linking reactions. The inefficient reaction and the use of DMSO as solvent raises the question, if this synthesis protocol is really suited to produce hydrophobic pectin derivatives for food packaging films as proposed by the authors.
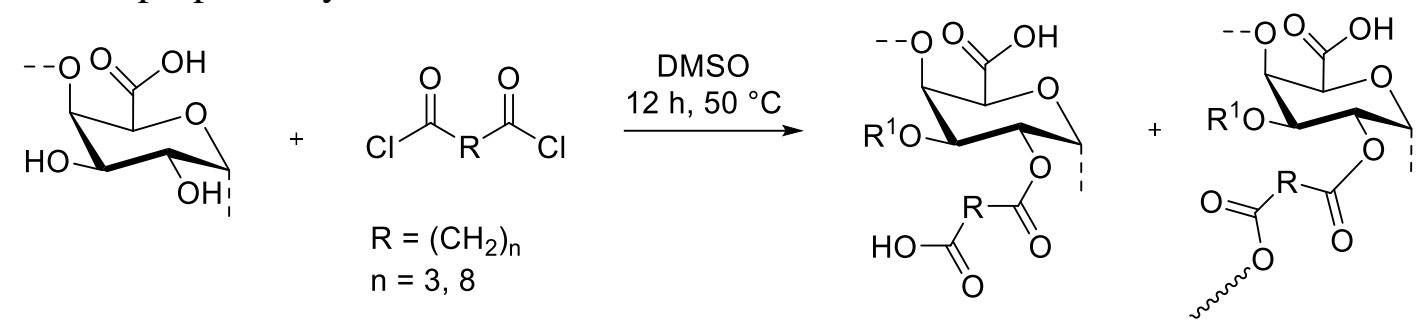

$$
\mathrm{R}^{1}=\mathrm{C}(\mathrm{O}) \mathrm{RCOOH}, \mathrm{H}
$$

Scheme 14. Homogeneous acylation of pectin with diacyl halides in dimethyl sulfoxide (DMSO) at $50{ }^{\circ} \mathrm{C}$ for $12 \mathrm{~h}$

Expanding the functionality of pectin further, the PS was modified with thiol group bearing compounds, including thioglycolic acid (Sharma and Ahuja 2011). The esterification proceeded with an excess of thioglycolic acid in water aided by $\mathrm{HCl}$ as catalyst (Scheme 15). A biopolymer derivative with a thiol content of $0.6 \mathrm{mmol} / \mathrm{g}$ was obtained. This value can be approximated to a DS of 0.1. Neither elemental analysis nor NMR data are available for the product. The thiol groups have been quantified with an Ellman's test and the ester formation was analyzed by IR spectroscopy. The materials produced are still capable to form gels with $\mathrm{Ca}^{2+}$ and show an increased mucoadhesion time compared to the starting material.

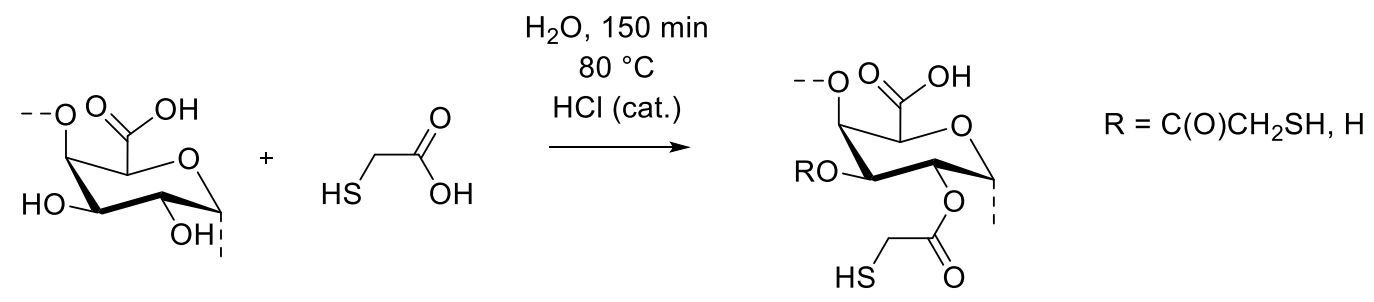

Scheme 15. Acylation of pectin with thioglycolic acid in acidic aqueous medium at $80^{\circ} \mathrm{C}$

The introduction of unsaturated ester groups in the pectin structure was achieved by Almeida et al. (2015). The reaction employed pectin in $N, N$-dimethyl formamide that was reacted with maleic anhydride at $70{ }^{\circ} \mathrm{C}$ for $24 \mathrm{~h}$ without a catalyst. 
The esterified pectin obtained bears maleate groups with a DS of up to 0.24 , as determined with ${ }^{1} \mathrm{H}$ NMR spectroscopy. The esterification increased the thermal stability of the pectin derivative, which started substantial degradation at $268{ }^{\circ} \mathrm{C}$. This temperature is $18{ }^{\circ} \mathrm{C}$ above the degradation temperature of the staring pectin. The material obtained showed an increased inhibition efficiency against Caco- 2 colon cancer cells compared to the starting pectin. In addition, the cytotoxicity of the PS was also lowered by this modification.

\section{Si-O Bond Formation}

The formation of Si-O bonds is a reaction closely related to acylation. The bonds formed can be inert depending on the neighboring substituents at the silicon atom due to steric hindrance or easily cleavable, e.g., by acid-catalyzed hydrolysis. A condensation reaction of pectin with 3-aminopropyltriethoxysilan (APTES) in toluene was reported, resulting in an amino group bearing biopolymer derivative (Baran 2019). The resulting nucleophilic pectin derivative was further treated with thiophene-1carbaldehyde to form a Schiff base. This imine structure was employed as a ligand for a palladium complex, which could be applied for efficient Suzuki-Miyaura reaction (Scheme 16). The catalyst could be used without activity loss for 12 catalytic cycles. The Pd-loaded pectin derivative (14 w\% Pd) showed no leaching of the metal.

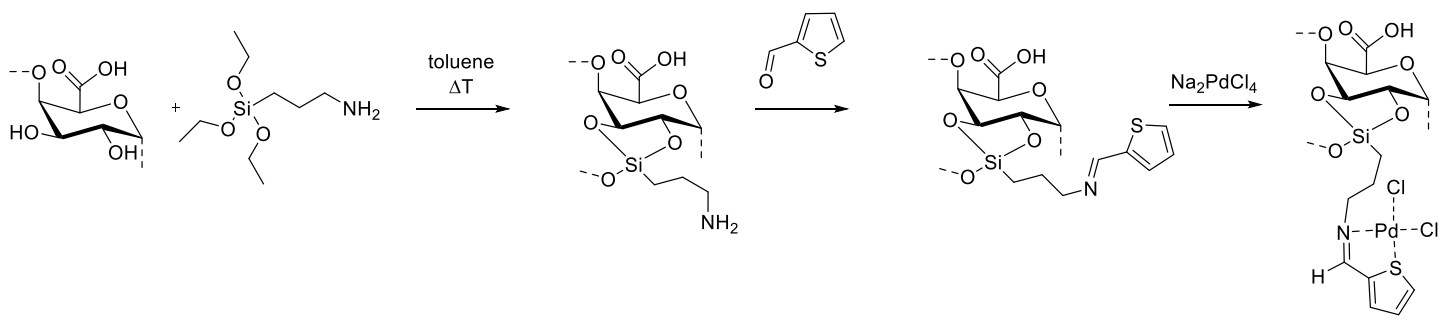

Scheme 16. Condensation of 3-aminopropyltriethoxysilan with pectin, imine formation with thiophene-1-carbaldehyde, and Pd complexation led to a polysaccharide derivative fit for Suzuki-Miyaura reaction (adapted from Baran 2019)

Very recently, a homogeneous approach to prepare silylated pectin derivatives was published employing PGA in dry formamide (FA) with trimethylsilylchloride (TMSCl)/imidazole or 1,1,1,3,3,3-hexamethyldisilazane (HMDS) as reagent (Würfel et al. 2019a). The reaction was shown to be more efficient employing HMDS, leading to a trimethylsilyl polygalacturonic acid derivative with a DS of 1.8, on the one hand. On the other hand, TMSCl was found to be much less efficient, leading to a DS of 0.60 only (Scheme 17). A complete silylation of the hydroxyl groups was not feasible even with a large excess of reagent (12 eq.). The silylated pectin derivative obtained was prone to hydrolysis in water. Additionally, side reactions could be identified, which led on the one hand to cross-linking with TMSCl, and on the other hand to the formation of amide groups with HMDS during the reaction.

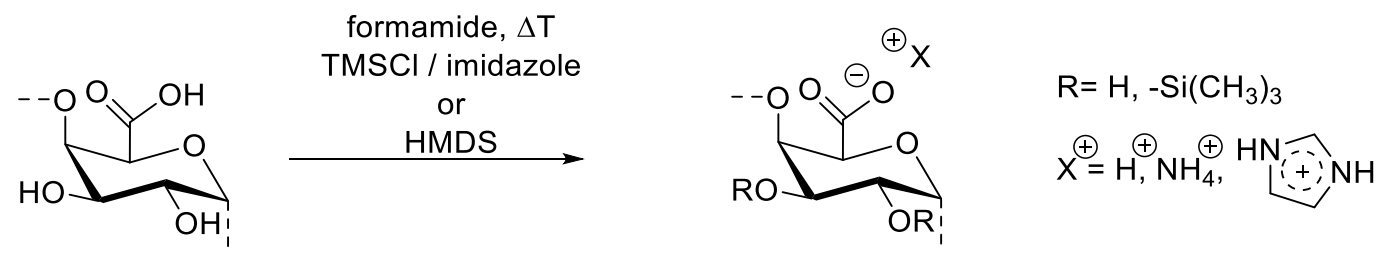

Scheme 17. Silylation of polygalacturonic acid with trimethylsilyl chloride (TMSCI)/imidazole or 1,1,1,3,3,3-hexamethyldisilazane (HMDS) in formamide resulting in trimethylsilyl polygalacturonic acid derivatives (adapted from Würfel et al. 2019a) 


\section{S-O-bond Formation}

The synthesis of pectin sulfate and their anticoagulant activity was studied (Vityazev et al. 2010). For the sulfation reaction of pectin, pyridine monomethyl sulfate, pyridine sulfur trioxide, and chlorosulfonic acid have been investigated (Scheme 18). All three sulfation reagents employed led to a partial PS degradation. The sulfating reagent with the best results considering degree of sulfation, degree of degradation, and product yield turned out to be the chlorosulfonic acid. The specific anticoagulant activity of the products obtained depends further on the plant species employed for pectin extraction, the composition of the pectin employed, and the degree of sulfation introduced. The specific antithrombin activity of the biopolymer derivatives obtained was assayed and showed to be superior in contrast to the starting pectin.

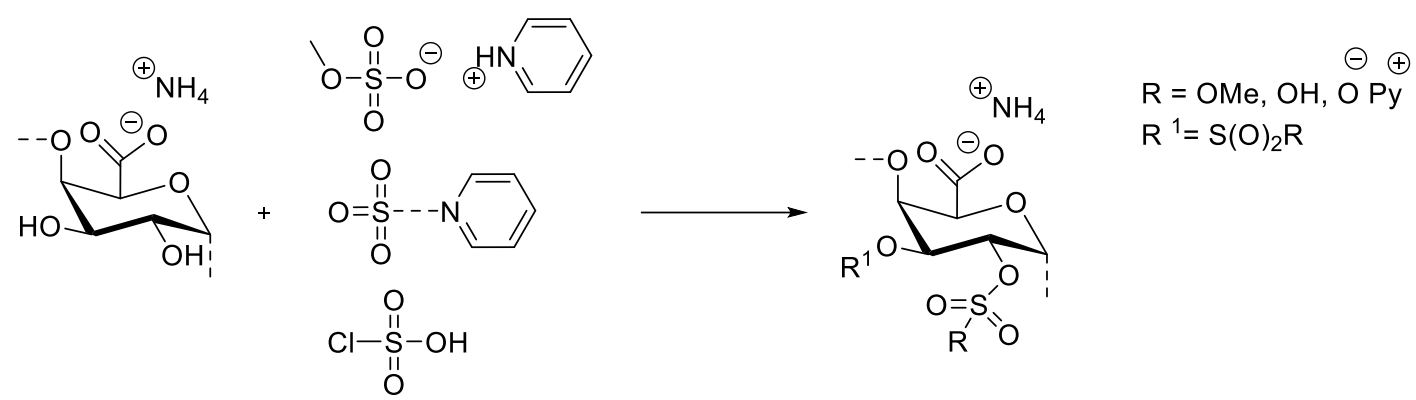

Scheme 18. Sulfation of pectin with pyridine monomethyl sulfate (top), pyridine sulfur trioxide (middle), and chlorosulfonic acid (bottom)

The anticoagulant activity of pectin sulfates has also been investigated by another group (Fan et al. 2011). The sulfating reagent to obtain pectin sulfate was trisulfonated sodium amine, which can be obtained by the reaction of $\mathrm{NaSO}_{3}$ and $\mathrm{NaNO}_{3}$ in water at $90{ }^{\circ} \mathrm{C}$. This path allows the sulfation of pectin in aqueous solution. The DS was determined after hydrolysis of the pectin sulfate with hydrochloric acid and transforming it into $\mathrm{BaSO}_{4}$. The amount of $\mathrm{BaSO}_{4}$ as homogeneous dispersion was determined with UV/Vis spectroscopy, at an absorption wavelength of $360 \mathrm{~nm}$. It is unclear how a homogeneous dispersion was maintained during the measurement and how this insoluble material was referenced against the water soluble $\mathrm{K}_{2} \mathrm{SO}_{4}$ employed for calibration. The literature cited for this method of sulfate determination does not describe the procedure employed (Schöniger 1956). Additionally, the calculation of the DS values given in the paper uses incorrect values for the molecular weight of the repeating unit. Thus, the DS values given are questionable and cannot be compared with other literature data.

\section{P-O-bond Formation}

A reaction of pectin with trisodium trimetaphospate in strongly basic aqueous medium $(\mathrm{pH}=12)$ resulted in pectin phosphate derivatives (Scheme 19, Souto-Maior et al. 2009). The pectin phosphates showed reduced water solubility due to crosslinking (Scheme 19). The polymer network obtained have been probed by FTIR spectroscopy and thermogravimetric analysis. The pectin phosphates could be casted in combination with an aqueous dispersion of poly(methyl methacrylate) into films at a temperature of $50{ }^{\circ} \mathrm{C}$. The films showed changes in water vapor permeability and hydration properties correlated to the pectin phosphate content. These films are potential drug release materials suitable for preventing the premature release of drugs in the upper part of the gastrointestinal tract. 

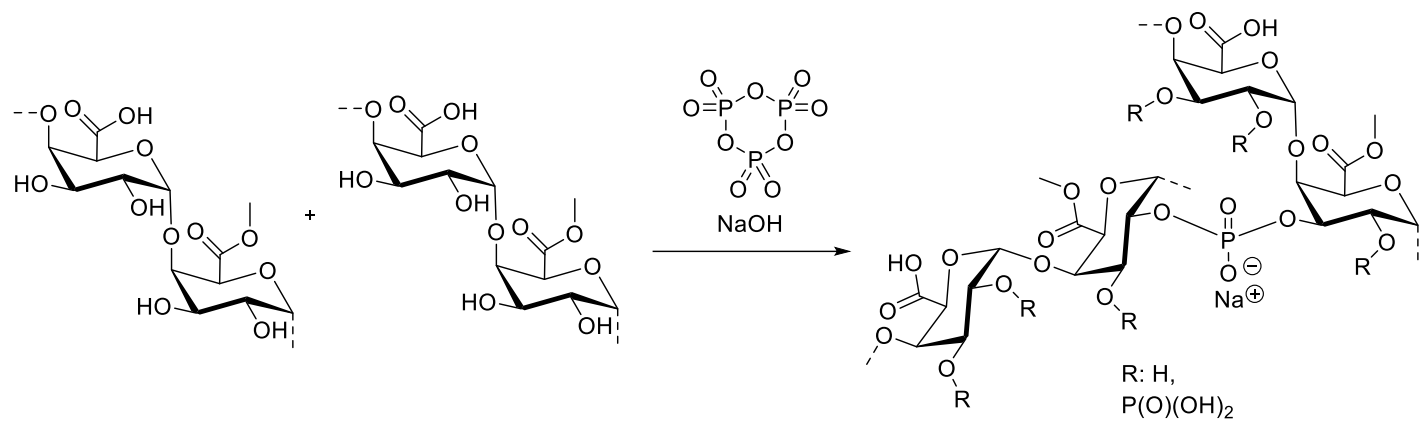

Scheme 19. Network formation of pectin and trisodium trimethaphosphate in basic aqueous solution (adapted from Souto-Maior et al. 2009)

\section{Alkylation Reaction at the Hydroxyl Group}

Alkylation of the hydroxyl groups of pectin is difficult to accomplish, due to the sensitive nature of pectin, which shows backbone degradation at $\mathrm{pH}$ values above 6.8 (Albersheim et al. 1960). Thus, a fast decrease in molecular weight occurs. The synthesis of quaternary ammonium derivatives of pectin employing (3-chloro-2hydroxypropyl)trimethylammonium chloride (CHPTAC) was reported in the presence of sodium hydroxide in water (Fan et al. 2012). The reaction is nonselective and leads to alkylation at the hydroxyl groups as well as esterification at the C6 carboxylate group (Scheme 20). This can be assumed, considering the DS obtained at high amounts of reagent (5 eq. and above, CHPTAC). The DS exceeds a value of 2 , which can only happen if the carboxylate groups react as well with the epoxide formed during the reaction (Fraenkel-Conrat and Olcott 1944). The quaternary ammonium pectin derivatives have been probed against their antibacterial and antifungal activities. Unfortunately, the materials synthesized showed no enhanced inhibition activity compared to the starting pectin.

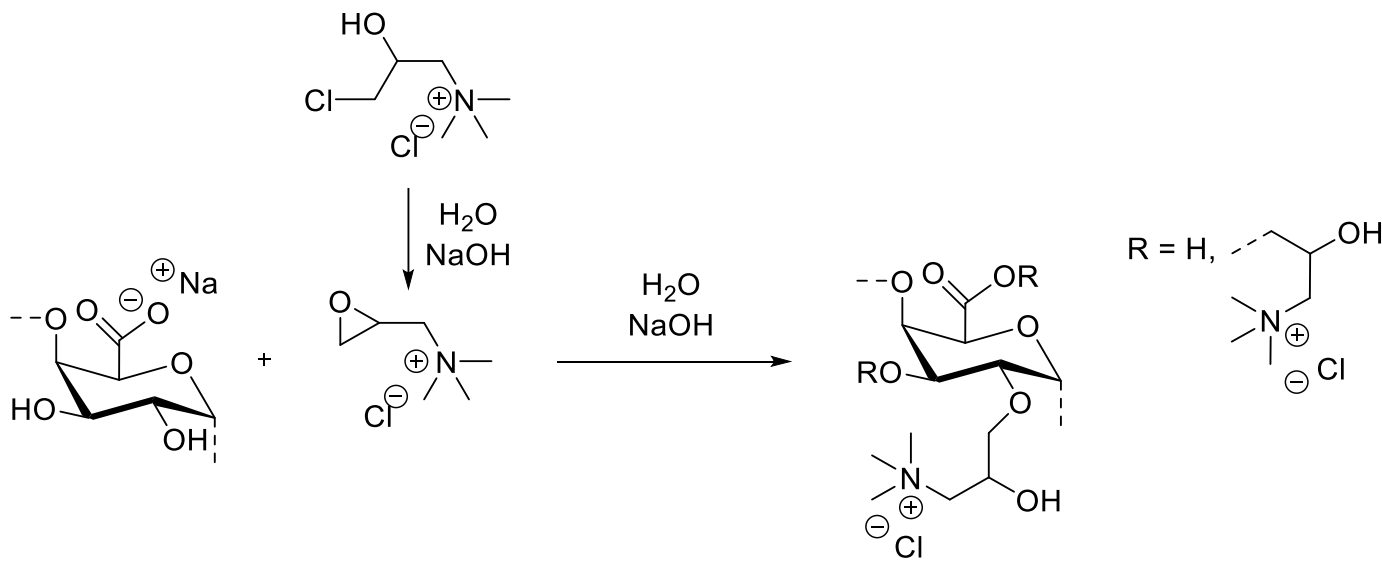

Scheme 20. Formation of ether derivatives of pectin by addition reaction of pectin with in situ generated glycidyltrimethylammonium chloride in basic aqueous medium (adapted from Fan et al. 2012)

A similar reaction was published by Bostanudin et al. (2019) describing the etherification of pectin in water at a $\mathrm{pH}$ value of 12 lasting for $16 \mathrm{~h}$ (Scheme 21). The DS ranging from values of 0.10 up to 0.14 was calculated from the ${ }^{1} \mathrm{H}$ NMR spectra. The possibility of a reaction at the carboxylic acid moiety was not described. 


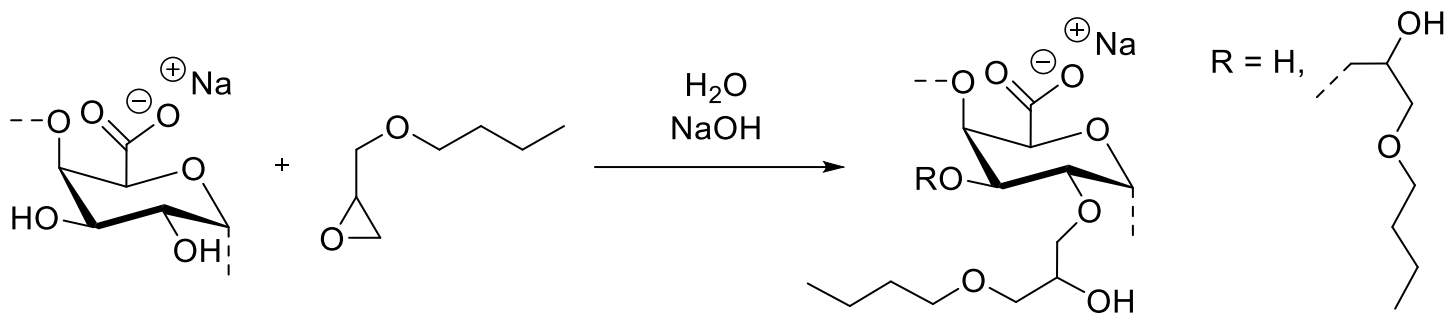

Scheme 21. Synthesis of hydrophobic pectin derivatives useful for nanoparticle formation and drug loading (adapted from Bostanudin et al. 2019)

The products formed have been characterized employing water and deuterium oxide as a solvent for gel permeation chromatography and ${ }^{1} \mathrm{H}$ NMR measurements, respectively. Confusingly, water could also be employed as dispersion phase in the precipitation of the material for the formation of NP. An influence of the drug incorporated seems likely to have changed the solubility of the system; however, it was not discussed further. The NP formed showed diameters of 250 to $280 \mathrm{~nm}$ with zeta potentials ranging from $-26 \mathrm{mV}$ to $-20 \mathrm{mV}$ (Fig. 5). The NP could incorporate the drug doxorubicin with up to $12 \mathrm{w} \%$.

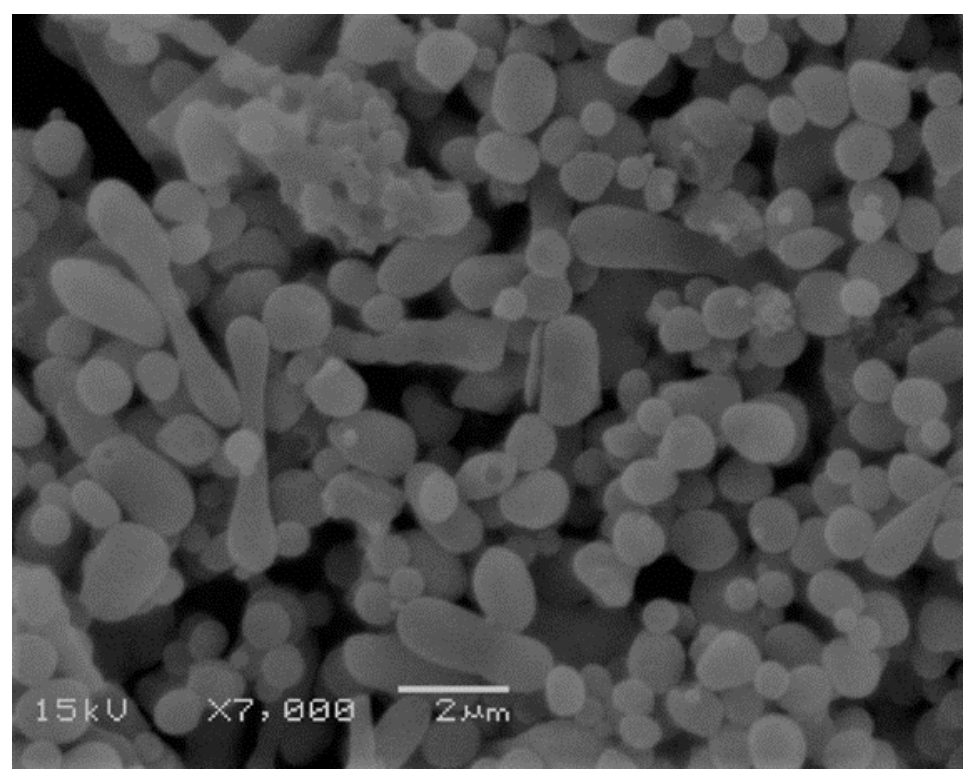

Fig. 5. Scanning electron microscopy image of lyophilized pectin ether particles (Polymers, 11(5), Bostanudin, M. F., Arafat, M., Sarfraz, M., Górecki, D. C. and Barbu, E., "Butylglyceryl Pectin Nanoparticles: Synthesis, Formulation and Characterization," p. 9, Copyright (2019)

The carboxymethylation of pectin was reported recently (Muthukumaran et al. 2018). The synthesis of carboxymethyl pectin (CMP) was performed in ethanol with aqueous $\mathrm{NaOH}$ and chloroacetic acid as reagent. The DS values were calculated by titration of the carboxylic acid moiety produced. Unfortunately, the formula employed to calculate the DS from the amount of $\mathrm{NaOH}$ was used without modification from a work employing carboxymethyl starch (Gao et al. 2011). Therefore, the carboxylic acid function already imparted in pectin was not accounted for. In addition, the formula for titration depicts the molecular mass of glucose and not galacturonic acid as repeating unit, rendering the data obtained incorrect. Additionally, no NMR spectroscopic analysis was performed to give clear insights to the CMP structure. The IR spectra were misinterpreted as well, accounting a signal at $2963 \mathrm{~cm}^{-1}$ in the pectin employed as a $\mathrm{COOH}$ vibration which disappears after basic treatment. Additionally, a signal at $1600 \mathrm{~cm}^{-1}$ in the resulting product was attributed to the $\mathrm{CH}_{2}$-group of the emerging 
carboxymethyl group. The more likely possibility was that the methyl ester moiety of the starting pectin gave the signal at $2963 \mathrm{~cm}^{-1}$, which was absent after basic treatment due to saponification. The new appearing signal at $1600 \mathrm{~cm}^{-1}$, which was not addressed in the cited work, likely corresponds to the carboxylate moiety formed. For these reasons, the values of carboxymethylation obtained cannot be compared with other carboxymethyl PSs.

\section{MISCELLANEOUS PECTIN DERIVATIVES OF INTEREST}

\section{Synthesis and Properties}

Solvents for pectin

Pectin as an ionic PS shows only a very limited solubility in organic solvents. Besides water, only FA is able to solubilize the compound at elevated temperatures. Mixtures of organic solvents and salts, e.g., DMSO/TBA fluoride or $N, N$ dimethylacetamide/LiCl, show no dissolution effect as well. A homogeneous solution is usually a key element for a complete PS transformation. Working with reactive compounds such as carboxylic acid anhydrides or -halides water can be discarded as solvent options, due to the significantly faster hydrolysis of the reagent in contrast to the acylation reaction with pectin. At best only small amounts of substitution are feasible with a large excess of acylation reagent employed working homogeneously in an aqueous medium. As a solvent, FA is not well suited for homogeneous pectin transformation either, due to side reactions occurring during a range of transformation reactions (Bredereck et al. 1959).

In the search for appropriate solvents suitable for modification reactions of pectin, it was found that the biopolymer is soluble in 1,3-dialkylimidazolium formats with up to $3 \mathrm{wt} \%$ (Fukaya et al. 2006). Later the commercially available ionic liquid (IL) 1-n-butyl-3-methylimidazolium (BMIM) chloride proved to be a fast solvent for PGA, the main component of pectin (Würfel et al. 2019b). Up to $5.3 \mathrm{wt} \%$ PGA can be dissolved by simple heating of the dried material in the IL for a short time at $140{ }^{\circ} \mathrm{C}$ (Scheme 22).
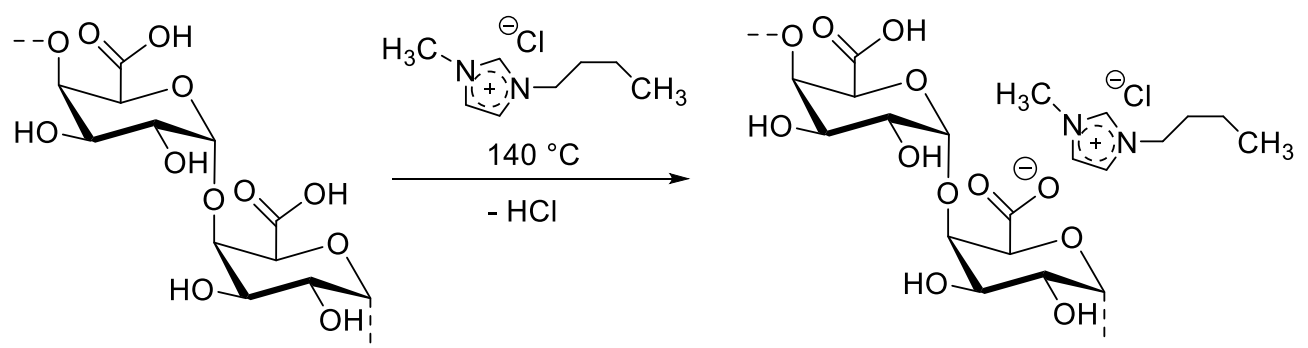

Scheme 22. Dissolution of polygalacturonic acid in 1-n-butyl-3-methylimidazolium chloride at $140^{\circ} \mathrm{C}$ partially leading to formation of a $1-n$-butyl-3-methylimidazolium polygalacturonate (adapted from Würfel et al. 2019b)

IR and NMR investigations showed that the solvent reacts with the PS at these temperatures, forming a BMIM-polygalacturonate with half of the carboxylic acid groups still present (Fig. 6). The IL can therefore be characterized as a derivatizing solvent for PGA. The degradation of the PS during the dissolution is negligible.

\section{Oxidation of pectin}

A systematic investigation about the oxidation of pectin with periodate at the vicinal hydroxyl groups at the $\mathrm{C} 2-\mathrm{C} 3$ position of the repeating unit was reported (Gupta et al. 2013). The influence of the reaction medium, $\mathrm{pH}$ value, temperature, and 
concentration of sodium periodate was varied to achieve a maximum degree of aldehyde groups (Scheme 23). The study shows that a solvent mixture of water/ethanol $80 / 20$ gave the highest amounts of aldehyde without precipitation of the pectin employed. Additionally, decreasing the $\mathrm{pH}$ value from 6.2 to 2.4 increases the formation of aldehyde groups from 0.8 to $3.6 \mathrm{mmol} / \mathrm{g}$. As expected, an increase in concentration of sodium periodate and reaction time yields an increase in aldehyde group formation as well. The reaction is accompanied by a decrease in the intrinsic viscosity of the pectin oxidized, most likely due to PS degradation.

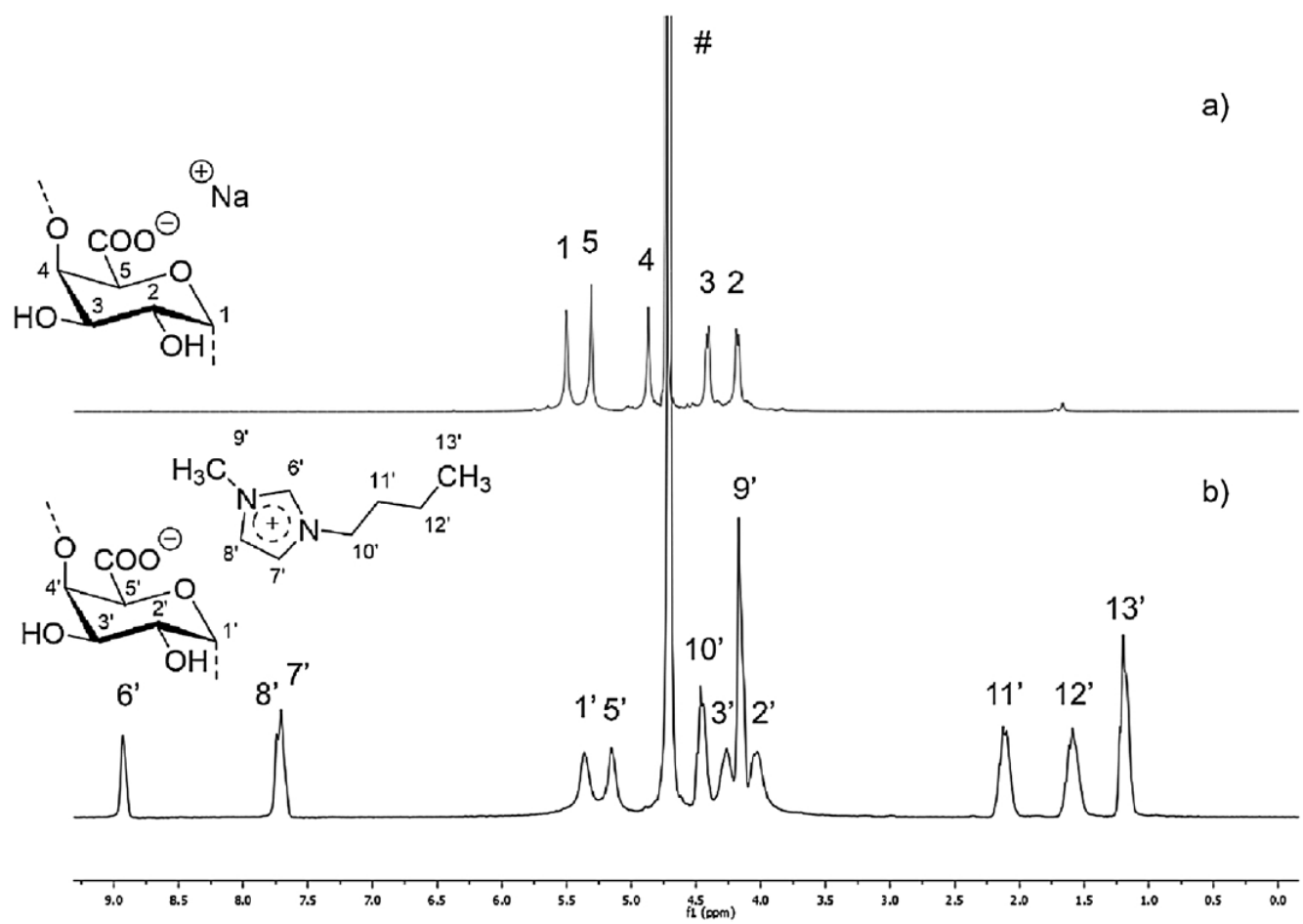

Fig. 6. ${ }^{1} \mathrm{H}$ NMR spectra of a) sodium polygalacturonate and b) 1-n-butyl-3-methylimidazolium polygalacturonate, measured in " $\mathrm{D}_{2} \mathrm{O}$. Reprinted from Carbohydrate Polymers, 207, Würfel, $\mathrm{H}$., Kayser, M., and Heinze, T., "Non-aqueous solvent for efficient dissolution of polygalacturonic acid," p. 793, Copyright (2019), with permission form Elsevier<smiles>COC(=O)C1(C(=O)O)C(O)OCC(O)C1O</smiles><smiles>O=C(O)C1C(O)C(O)C(O)C(O)C1O</smiles>

$$
\mathrm{H}_{2} \mathrm{O} / \mathrm{EtOH}
$$

$10-240 \mathrm{~min}$

$\mathrm{pH}: 2$ - 8<smiles>COC(=O)C1(C(=O)O)C(O)OC(O)C(O)C1O</smiles>

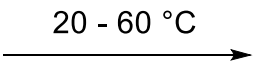<smiles>COC(C(=O)O)C(C=O)OC(C)C</smiles>

Scheme 23. Oxidation of pectin with sodium periodate in water/ethanol mixtures forming aldehyde groups (adapted from Gupta et al. 2013)

Periodate-oxidized pectin was employed to aid water solubility for amphotericin B (Kothandaraman et al. 2017). The amino group of this polyene macrolactone reacted with the aldehyde groups of periodate oxidized pectin, forming an imine. To further increase the stability of the linked drug, the imine was subsequently reduced to an amine employing sodium borohydride (Scheme 24). Both the imine and 
the amine derivative have been tested for their anti-fungal activity against Candida albicans and Aspergillus fumigatus. Furthermore, the antileishmanial activity was investigated for both compounds, showing that they are active against Leishmania donovani LV9 and DD8. The amine form showed to be a more active compound compared to the imine form.<smiles>COC(=O)C1C(O)C(O)C(OC(C=O)C(C=O)OC(C)C=O)C(O)C1C(=O)O</smiles>
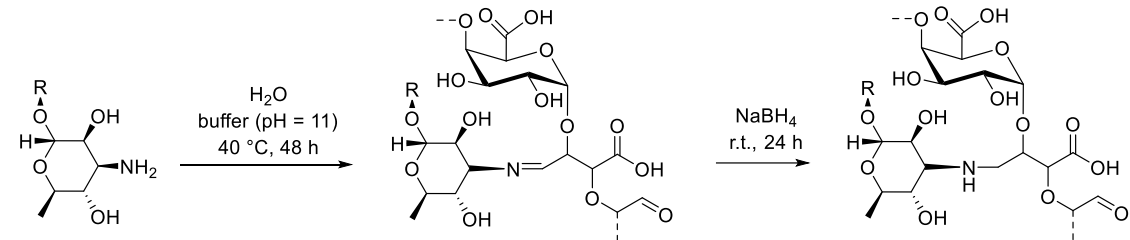

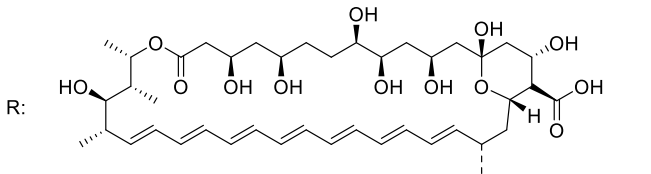

Scheme 24. Periodate oxidized pectin was employed in the covalent linkage of amphotericin B via an imine formation. Subsequent reduction of the imine with sodium borohydride led to the formation of an amine.
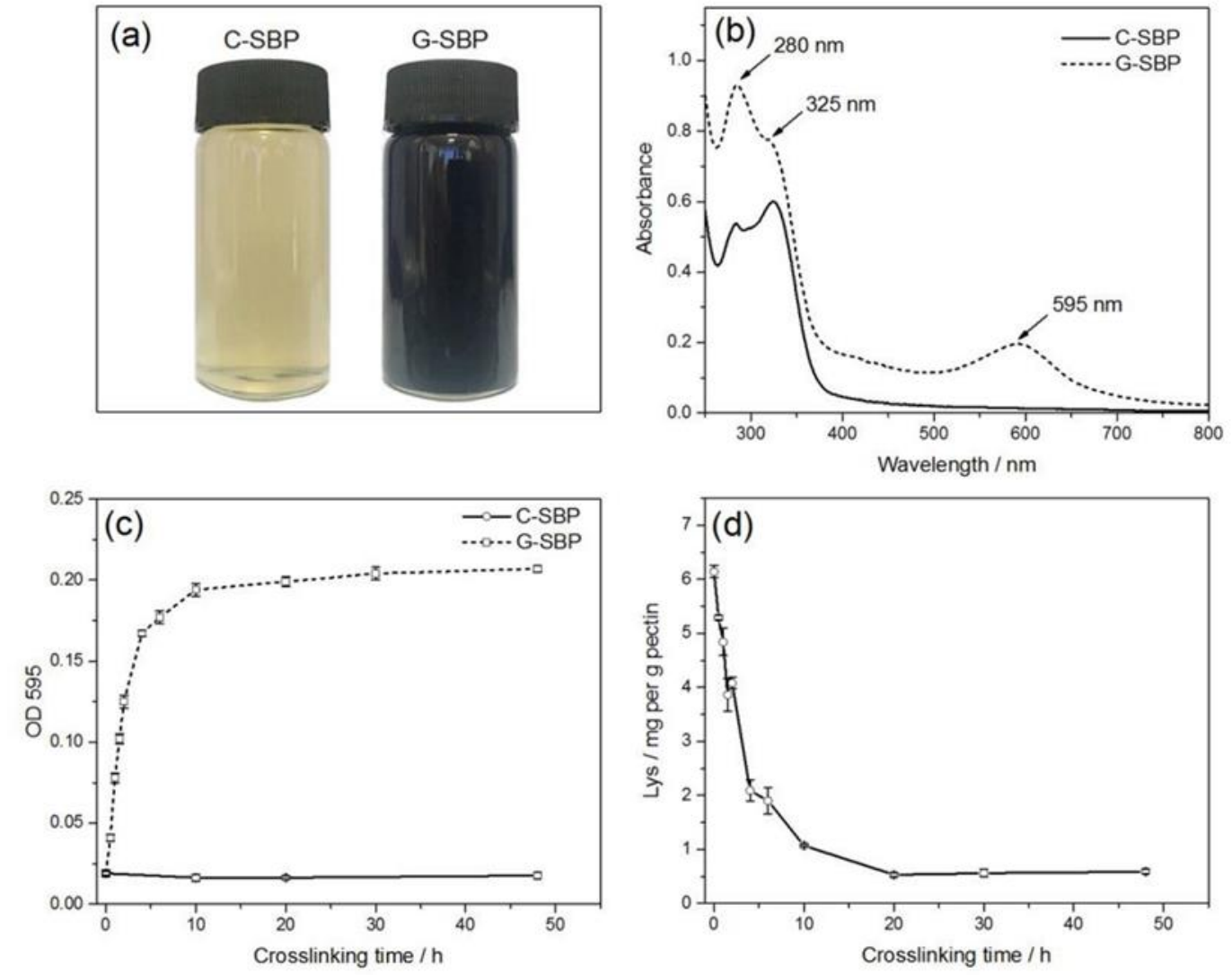

Fig. 7. Cross-linking reaction of amino groups with genipin results in a deep blue coloration of the cross-linked gel (a), which shows a new absorption band at $596 \mathrm{~nm}$. The cross-linking is finished within $10 \mathrm{~h}$ of reaction time which could be monitored with UV/Vis-spectroscopy (c) or determination of the free amino acid content (d). Reprinted from Food Hydrocolloids, 101, Lin, J., Yu, S., Ai, C., Zhang, T., and Guo, X., "Emulsion stability of sugar beet pectin increased by genipin crosslinking," p. 3, Copyright (2020) with permission from Elsevier

An interesting new cross-linker was employed in small amounts to enhance the molecular weight of native pectin (Lin et al. 2020). The cross-linker genipin, a 
hydrolysis product of gardenoside isolated from Gardenia jasminoides, can react with amino groups of protein impurities in native pectin, leading to an increase in molecular mass of the material and subsequently to a gel formation. Due to the formation of an aromatic system during cross-linking, the material starts to exhibit a blue coloration when reacted (Fig. 7, Butler et al. 2003). It must be mentioned that the cross-linking occurs exclusively with the protein impurities and genipin. Pectin is embedded in the genipin cross-linked network.

The products showed a decrease in their emulsion forming activity, but the emulsion stability was greatly increased compared to the starting material. The linker can be considered as an alternative to glutaraldehyde or formaldehyde because of its low toxicity that is especially important in food industry and drug delivery applications (Scheme 25, Manickam et al. 2014).

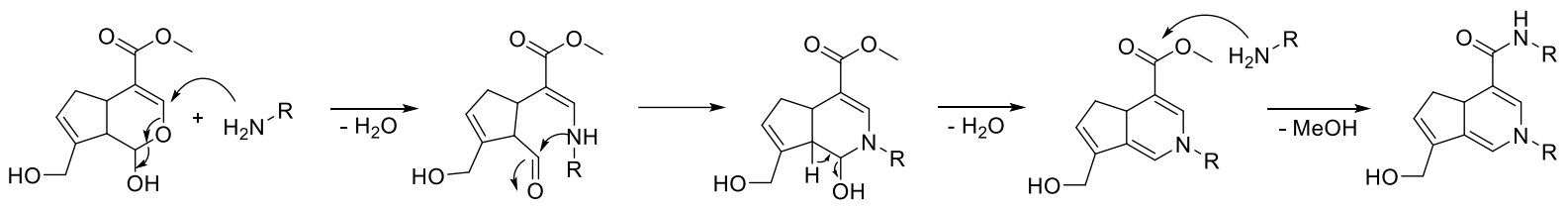

R: polysaccharide, amino acid

Scheme 25. Reaction of genipin with amino groups. Two reactions could be identified, which in combination lead to cross-linking and the deep coloration of the compounds. Adapted from Butler et al. 2003

\section{Energy Storage - Emerging Application Trends}

Lightweight materials for reversible energy storage are gaining much attention, due to changing energy generation techniques and fast increasing energy demands and storage necessities. The trend is most recognizable in the fields of electric cars and smart homes. Pectin, due to its water solubility and gelling properties depending on multivalent ions, was tested as an alternative material for electrolytes and as membrane material for applications in battery and fuel cell.

A blended material combining chitosan and pectin in a membrane for fuel cells was described (Cabello et al. 2017). Due to the opposite charges in the two PSs, an ionic induced cross-linking and compacting led to membrane materials that show smaller pore sizes compared to the pristine materials. The methanol permeability of the formed membranes was lowered as well, and the ion transport was decreased by an order of one magnitude when reaching a weight composition chitosan: pectin of 1:1. The stability against oxidizing agents was tested as well, showing an increase for chitosan/pectin membranes compared to pristine chitosan.

Vahini et al. (2019) prepared pectin-based electrolytes with various ratios of $\mathrm{NaNO}_{3}$ with a solution casting technique using distilled water as a solvent. Minor changes $\left(1\right.$ to $\left.3 \mathrm{~cm}^{-1}\right)$ in the FTIR spectra between the samples have been interpreted as a complexation reaction; however, the scanning resolution of the IR spectrometer was not reported. The electrical properties showed a high ionic conductivity $\left(4.2 \times 10^{-6}\right.$ $\mathrm{S} / \mathrm{cm}$ ) with a ratio of $91 \mathrm{~m} \%$ pectin to $9 \mathrm{~m} \% \mathrm{NaNO}_{3}$. This pectin $/ \mathrm{NaNO}_{3}$ system has a higher ionic conductivity as pure pectin $\left(2.7 \times 10^{-9} \mathrm{~S} / \mathrm{cm}\right)$. These values of conductivity clearly show that pectin acts as binder only. However, the conductivity falls short on biopolymer/salt mixtures already reported (Smitha et al. 2005; Cabello et al. 2017).

A mixture of pectin and $\mathrm{LiCl}$ was tested as a membrane produced by solution casting, which showed high conductivity $\left(2.08 \times 10^{-3} \mathrm{~S} / \mathrm{cm}\right)$ at a molar ratio of $1: 1$ (Perumal et al. 2018). The membrane was applied for the construction of a primary lithium ion battery. The open-circuit voltage was found to be $1.93 \mathrm{~V}$. 
As a binder, pectin was evaluated to sustain volume changes of $\mathrm{Si}$ anodes in lithium ion batteries (Wang et al. 2020). Thus, poly(acrylic acid) (PAA) and pectin were separately dissolved in distilled water and mixed dropwise by adding the pectin solution to the stirred solution of PAA (Scheme 26). The resulting homogeneous mixture was deposited on a Teflon surface and dried for one day to obtain a film. A three-dimensional network was formed based on the interactions of hydrogen bonds between the respective functional groups of pectin and PAA. This material showed a strong interfacial adhesion and a rapid electrolyte permeation and could be serve as efficient binder material for electrolytes.

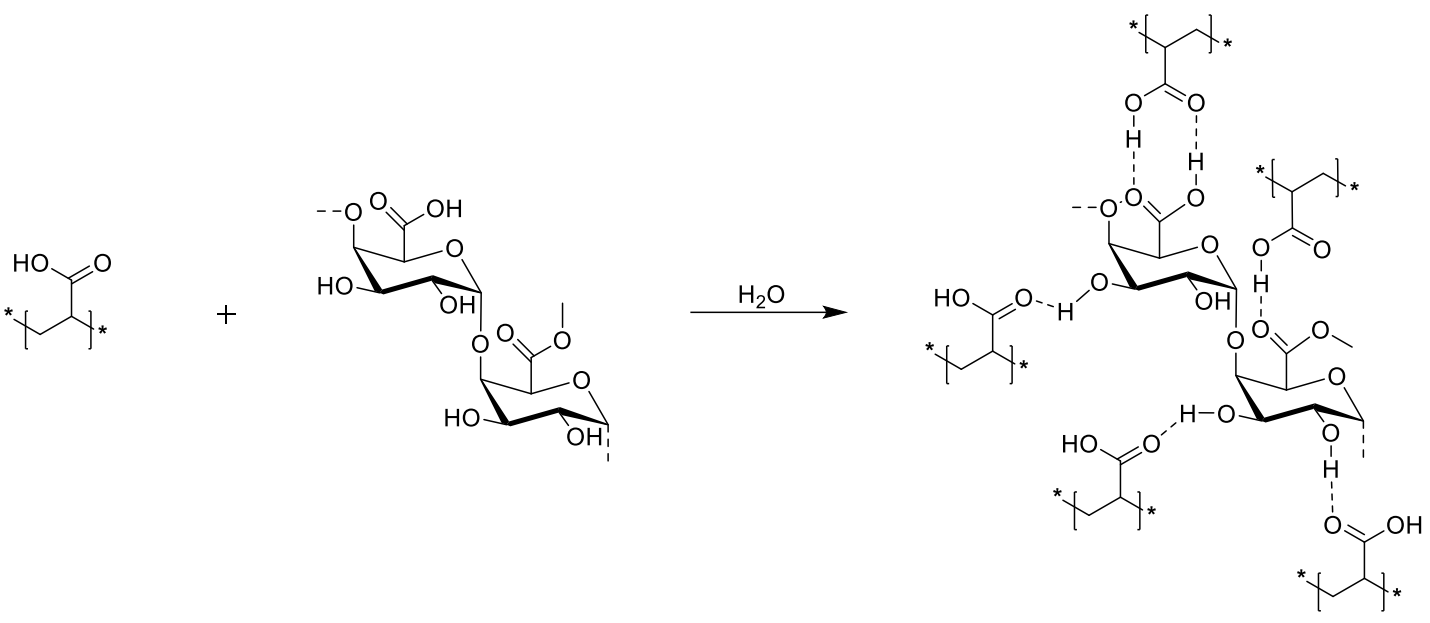

Scheme 26. Pectin/poly(acrylic acid) films as binder materials for electrolytes (adapted from Wang et al. 2020)

\section{HYDROGELS}

A topic of increasing research interest for applying cross-linked pectin derivatives is the field of hydrogels. Hydrogels are three-dimensional structures that have a high water absorbing capacity. They are found to be of great applicability in medical and pharmaceutical research and applications. Due to their interesting properties, such as anti-inflammatory, non-toxicity, non-irritating action, water and oxygen permeability, drug leaching, and biodegradability, they can be tailored to fit a specific application (Fu and in het Panhuis 2019). Hydrogels can be designed to exploit either chemical cross-linking, via covalent bonds, or physical cross-linking via ionic interactions, van der Waals, hydrophobic or hydrogen bond interactions (Hennink and van Nostrum 2012). LMP, pectin, and pectin hydrazide are known to form complexes with metal ions, and their three-dimensional network is described as an "egg box" model (Wang et al. 2019). The "egg box" model assumes that the carboxyl groups allow the binding of metal ions via ionic interactions (Cao et al. 2020). Physically crosslinked pectin gels form reversible bonds that are less stable than covalent ones. Nevertheless, this cross-linking method is of great interest, especially for the encapsulation of bioactive compounds and for the removal of metal ions from wastewater (Wang et al. 2019; Hennink and van Nostrum 2012). Covalent linking of pectin derivatives is more useful in tissue engineering and in medical adhesion applications, due to their higher stability and better mechanical properties. In the following section, the hydrogel formation has been divided into permanent and potentially reversible cross-linking. The reversibility can generally be triggered by a $\mathrm{pH}$ response or a reduction reaction. 


\section{Permanent Cross-linking}

Acyl hydrazide cross-linking

A hydrogel was prepared by reacting EDC activated PGA with adipic acid dihydrazide (ADH) (Peng et al. 2011). Subsequent activation of a short-chain hyaluronate (sHA) with 1,1'-carbonyldiimidazole (CDI) and a second acyl hydrazide formation with the PGA dihydrazide led to the PGA-ADH derivative (Scheme 27). The modification of sHA with CDI was carried out in FA at RT. Both derivatives were characterized by ${ }^{1} \mathrm{H}$ NMR spectroscopy. A mixing of the aqueous solution yields crosslinking and formation of the hydrogel, which has a high water content and a great compressive modulus. Additionally, a membrane was prepared from the hydrogel that was applied in animal implant studies, where it exhibited good anti-adhesion properties.<smiles>COC(=O)C1(C(=O)O)C(O)C(O)C(O)C(O)C1C(=O)NNC(=O)CCCCC(=O)NN</smiles>

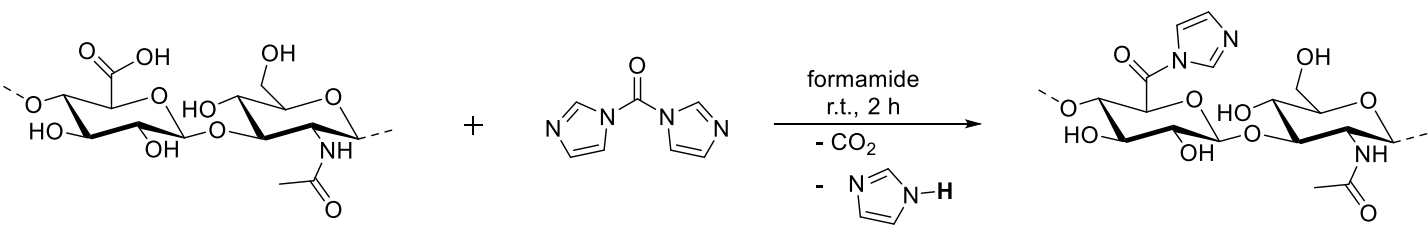

Scheme 27. Activation of pectin with 1-ethyl-3-(3-dimethylaminopropyl)carbodiimide (EDC) in water and reaction with adipic acid dihydrazide forming a bisacyl hydrazide structure (top). Formation of an activated hyaluronic acid derivative with 1,1'-carbonyldiimidazole in formamide at RT (bottom)

\section{Acyl hydrazone cross-linking}

An acyl hydrazide could be applied as the starting structure to generate crosslinked pectin derivatives (Lin et al. 2015).
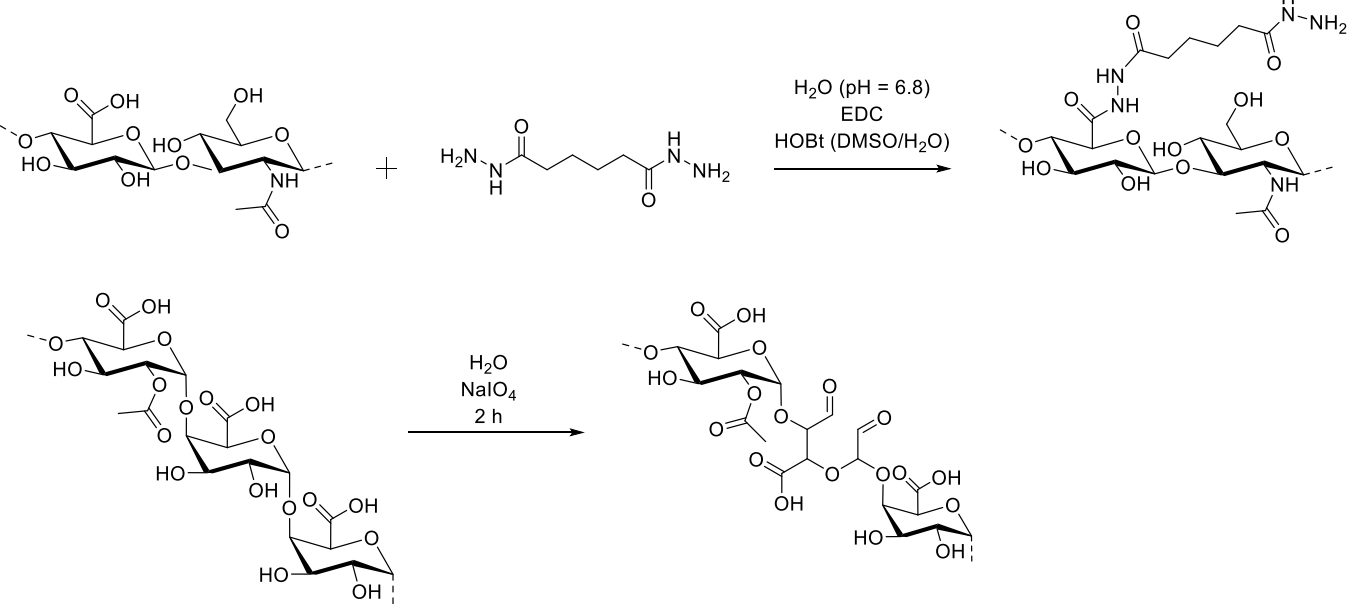

Scheme 28. Activation of hyaluronic acid with 1-ethyl-3-(3-dimethylaminopropyl)carbodiimide (EDC) and subsequent reaction with adipic acid dihydrazide (top). Oxidation of pectin with sodium periodate in water leading to aldehyde groups bearing pectin (bottom, adapted from Lin et al. 2015) 
Thus, hyaluronic acid (HA) was allowed to react in water with EDC and subsequently with ADH. This HA dihydrazide derivative was further treated with aldehyde containing oxidized polygalacturonic acid (PGA-CHO) in a phosphate buffer solution (Scheme 28). Due to the formation of acyl hydrazone, PGA-HA gel is formed very rapidly, which shows a swelling ratio of $18.4 \%$ to $22.2 \%$, depending on mixing ratio of the two components. The degree of swelling varies with the strength of crosslinking. The hydrogel was also probed in animal implant studies, and tests confirmed that the PGA-HA gel is slower in its degradation within 1 week than HA and the FDA approved Medishield $^{\mathrm{TM}}$, which was also employed for the tests. Furthermore, the area of scar tissue formed after a surgical intervention was lower for the Medishield ${ }^{\mathrm{TM}}$ $\left(0.41 \pm 0.11 \mathrm{~mm}^{2}\right)$ and PGA-HA $\left(0.30 \pm 0.10 \mathrm{~mm}^{2}\right)$ gels than compared with saline solution $\left(0.51 \pm 0.14 \mathrm{~mm}^{2}\right)$ and a HA $\left(0.55 \pm 0.09 \mathrm{~mm}^{2}\right)$ gel.

Further studies showed that loading of the PGA-HA gel with bioactive substances, e.g. ibuprofen (IBU), is possible (Lin et al. 2016). Therefore, the carboxyl group of IBU was activated with $N, N^{\prime}$-dicyclohexylcarbodiimide and transformed into an activated ester employing $N$-hydroxysuccinimide. The active ester reacted with hydrazide groups at the modified pectin forming bisacyl hydrazide derivatives with the IBU. The hydrogels were tested as wound dressing after surgical intervention. The drug loaded compound PGA-HA-IBU showed also reduced scar tissue formation $(0.91 \pm 0.33$ $\left.\mathrm{mm}^{2}\right)$ slightly superior to an unloaded PGA-HA $\left(0.97 \pm 0.34 \mathrm{~mm}^{2}\right)$ hydrogel and more efficient than pure IBU $\left(1.29 \pm 0.31 \mathrm{~mm}^{2}\right)$ and saline solution $\left(1.04 \pm 0.21 \mathrm{~mm}^{2}\right)$.

\section{Radical cross-linking}

Chemical cross-linking via radical polymerization is described by Khan et al. (2019). In this study, pectin was converted with the monomer 2-acrylamido-2methylpropan sulfonic acid in the presence of ammonium persulfate and sodium metabisulfite as redox initiator. Methylene bisacrylamide was used as a cross-linking agent to obtain a three-dimensional network. The gels were prepared by dissolving each substance separately. Then the aqueous solutions were mixed and heated to $60{ }^{\circ} \mathrm{C}$ for $24 \mathrm{~h}$. The hydrogels formed were tested for drug loading with captopril, an angiotensinconverting enzyme inhibitor, and exhibited a great mechanical strength. The best swelling ratio was obtained at a $\mathrm{pH}$ value of 7.4 and the release of captopril took place at a $\mathrm{pH}=1.2$. Both drug release and swelling decreased with an increasing concentration of cross-linking agents.

\section{Reversible Cross-linking}

Disulfide cross-linking

A recent study of chemical cross-linking employed the amide formation of cysteine with the $\mathrm{C} 6$ carboxylic acid function of PGA. The cysteine moiety can crosslink via oxidation of the thiol group attached forming disulfides (Peng et al. 2013). This cross-linking reaction is $\mathrm{pH}$-sensitive, allowing disulfide formation above a $\mathrm{pH}$ value of 8.5 only. The hydrogels obtained show a very low degree of swelling of $0.8 \%$ and a high gel content, which is a basic requirement for biomaterials used as anti-adhesion device. The degree of swelling increases as the degree of cross-linking decreases. Due to the reversible nature of this covalent cross-link, it is of interest for drug delivery systems as well (Saito et al. 2003).

\section{Schiff base cross-linking}

Gelatin was used to cross-link nanofibers of aldehyde containing HMP, which was prepared by periodate oxidation in water (Shi et al. 2020). The cross-linking occurred, due to a Schiff base reaction. The nanofibers were placed in a gelatin solution 
(ethanol/water, 70:30) and reacted at $37{ }^{\circ} \mathrm{C}$ for $24 \mathrm{~h}$. The chemical cross-linking was confirmed by IR spectroscopy. After the formation of imine cross-links, the nanofibers exhibit a larger diameter and a rougher surface as ascertained by use of SEM measurements (Fig. 8). The cross-linked nanofibers promote cell infiltration due to the hydrophilicity and cell affinity of gelatin and can potentially be used for the regeneration of soft tissues such as skin or cartilage.
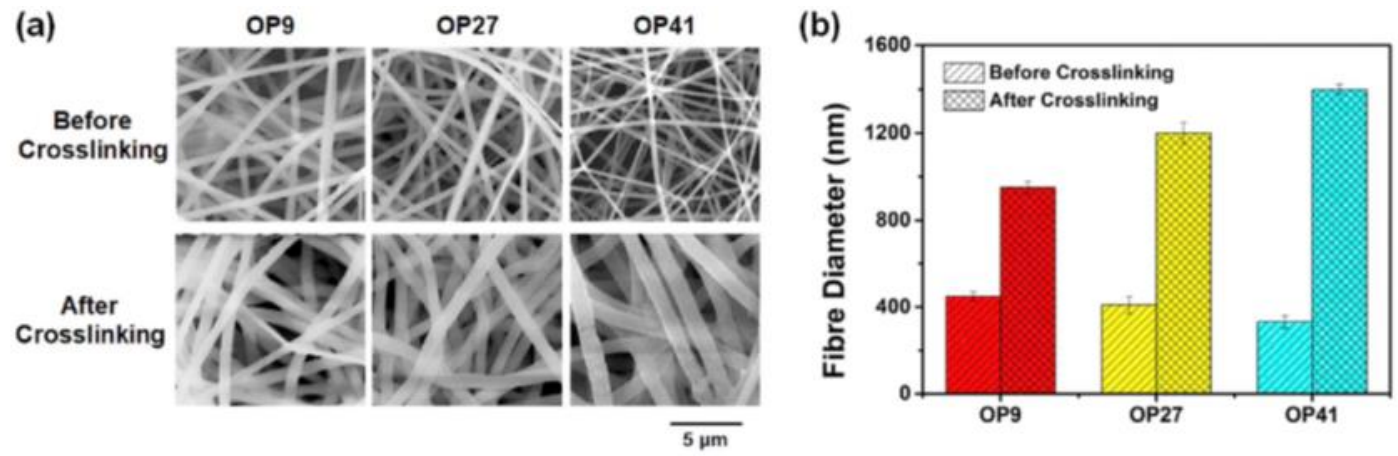

Fig. 8. Scanning electron microscopy images and diameter of electrospun oxidized pectin fibers before and after gelatin cross-linking. Reprinted from Materials Science and Engineering: C, 112, Shi, X., Cui, S., Song, X., Rickel, A. P., Sanyour, H. J., Zheng, J., Hu, J., Hong, Z., Zhou, Y., and Liu, Y. "Gelatin-crosslinked pectin nanofiber mats allowing cell infiltration," p. 3, Copyright (2020), with permission from Elsevier

\section{CONCLUDING REMARKS}

Pectin, as a very important yet underestimated biopolymer resource, has been becoming more popular in the last decade. The bi-functional nature of the $\alpha$-1,4-linked anhydrogalacturonic acid repeating unit allows for a wider range of one-step transformations compared to other PSs, such as cellulose or starch, which are mainly used today resulting in a much broader spectrum of possible functional materials. Some properties of pectin, including its ionic nature, can lead to unpredictable behavior such as reactivity and solubility. The ionic nature leads to water solubility, which raises opportunities in medical and food applications, on the one hand. On the other hand, it interferes with the solubility in organic solvents, the preferred medium for efficient derivatization reactions in the laboratory. Another example is the inherent degradability of pectin and most of its derivatives. One the one hand, this is a desired property considering the raising concern of micro-plastic pollution. On the other hand, this is a drawback if long life stable products are required. A general trend for pectin modification can be found leading away from homogeneous transformation reactions to heterogeneous processes, which are commonly required in industrial applications, considering the reaction efficiency and the cost and the recycling of solvents.

However, a concerning issue in a large number of papers presented is the use of incorrect chemical terms, which can lead to great confusion. This state of affairs may prohibit a clear communication of research results among scientist and prevents a direct comparison of research work. These trends have even been adapted in review articles published (Cheng et al. 2015). From a chemist's point of view, there is a clear differentiation between an alkylation- and acylation reactions. The definitions for both types of reaction are found in the IUPAC goldbook and should be used stringently (Moss et al. 1995). Another term, frequently employed to circumvent the terms alkylation and acylation altogether is the term conjugation, which also has a completely different meaning in chemistry and therefore should be avoided as well (Muller 1994). 
In addition to the problems of terminology, a number of studies refrain from characterizing the pectin employed in their research adequately. The focus is directed on the effects obtained by a modification at times. It is desirable to develop a distinct structure-property relationship; thus a detailed structure characterization is highly relevant in further studies about pectin and pectin derivatives. Due to the fact that pectin is rather complex, at least the galacturonic acid content, the molecular weight, and the degree of esterification of the starting pectin should be stated (Müller-Maatsch et al. 2014). Otherwise, experiments performed and results obtained are hardly reproducible, in particular by other researchers.

Nevertheless, the increase in the number of publications concerning the chemical modification of pectin, PGA, and PSs in general reflects a global trend to intensify their usability in applications, as they are the largest group of available bioresources and will contribute to substitute for petrochemical based materials. Additionally, this research aids in developing more sustainable industrial processes, and helps to reduce the concerns about the environmental problems based on chemistry in general.

It is the authors' hope that the review helps to foster the development of novel derivatives of pectin and PGA by chemical modification in order to take full advantage of this very important renewable resource. The critical issues discussed are not only true for pectin but also for other PSs. Thus, it is highly desirable that the chemistry of this most important class of renewable resources will use the terminology of organic chemistry adequately. Of particular interest is the clear structure determination of the natural products due to the fact that they may differ in structure depending on the source and the isolation procedure.

\section{ACKNOWLEDGEMENT}

The manuscript was financially supported by the Deutsche Forschungsgemeinschaft (DFG, German Research Foundation) - project number 316213987 SFB 1278 (project A02)

\section{REFERENCES CITED}

Albersheim, P., Neukom, H., and Deuel, H. (1960). "Splitting of pectin chain molecules in neutral solutions," Archives of Biochemistry and Biophysics 90, 4651. DOI: 10.1016/0003-9861(60)90609-3

Almeida, E. A. M. S., Facchi, S. P., Martins, A. F., Nocchi, S., Schuquel, I. T. A., Nakamura, C. V., Rubira, A. F., and Muniz, E. C. (2015). "Synthesis and characterization of pectin derivative with antitumor property against Caco- 2 colon cancer cells," Carbohydrate Polymers 115, 139-145. DOI: 10.1016/j.carbpol.2014.08.085

Aris, Z. F. M., Bouldin, R. M., Pelletier, M. G. H., Gaines, P., Budhlall, B., and Nagarajan, R. (2017). "Microwave-assisted synthesis and characterization of hydrophilically functionalized polygalacturonic acid," Carbohydrate Polymers 155, 432-439. DOI: 10.1016/j.carbpol.2016.08.070

Bae, I. Y., Rha, H. J., Lee, S., and Lee, H. G. (2011) "Preparation and characterization of pectin hydroxamates from citrus unshiu peels," Journal of Excipients and Food Chemistry 2(3), 89-94. 
Baran, T. (2019). "Highly recoverable, reusable, cost-effective, and Schiff base functionalized pectin supported Pd(II) catalyst for microwave-accelerated Suzuki cross-coupling reactions," International Journal of Biological Macromolecules 127, 232-239. DOI: 10.1016/j.ijbiomac.2019.01.046

Bostanudin, M. F., Arafat, M., Sarfraz, M., Górecki, D. C., and Barbu, E. (2019). "Butylglyceryl pectin nanoparticles: Synthesis, formulation and characterization," Polymers 11(5), 789. DOI: 10.3390/polym11050789

Bredereck, H., Gompper, R., Rempfer, H., Klemm, K., and Keck, H. (1959). "Formamid-Reaktionen, XII. Alkylierungs und Acylierungsreaktionen an Formamid: Tris-formylamino-methan," Chemische Berichte 92(2), 329-337. DOI: 10.1002/cber.19590920213

Butler, M. F., Ng, Y.-F., and Pudney, P. D. A. (2003). "Mechanism and kinetics of the crosslinking reaction between biopolymers containing primary amine groups and genipin," Journal of Polymer Science Part A: Polymer Chemistry 41(24), 39413953. DOI: 10.1002/pola.10960

Cabello, S. D. P., Ochoa, N. A., Takara, E. A., Mollá, S., and Compañ, V. (2017). "Influence of pectin as a green polymer electrolyte on the transport properties of chitosan-pectin membranes," Carbohydrate Polymers 157, 1759-1768. DOI: 10.1016/j.carbpol.2016.11.061

Calce, E., Bugatti, V., Vittoria, V., and Luca, S. D. (2012). "Solvent-free synthesis of modified pectin compounds promoted by microwave irradiation," Molecules 17, 12234-12242. DOI: 10.3390/molecules 171012234

Calce, E., Mignogna, E., Bugatti, V., Galdiero, M., Vittoria, V., and Luca, S. D. (2014). "Pectin functionalized with natural fatty acids as antimicrobial agent," International Journal of Biological Macromolecules 68, 28-32. DOI: 10.1016/j.ijbiomac.2014.04.011

Cao, L., Lu, W., Mata, A., Nishinari, K., and Fang, Y. (2020). "Egg-box model-based gelation of alginate and pectin: A review," Carbohydrate Polymers 242, 116389. DOI: 10.1016/j.carbpol.2020.116389

Chen, J., Liu, W., Liu, C.-M., Li, T., Liang, R.-H., and Luo, S.-J. (2015). "Pectin modifications: A review," Critical Reviews in Food Science and Nutrition 55(12), 1684-1698. DOI: 10.1080/10408398.2012.718722

Chen, J., Ye, F., Zhou, Y., and Zhao, G. (2018), “Thiolated citrus low-methoxyl pectin: Synthesis, characterization and rheological and oxidation-responsive gelling properties," Carbohydrate Polymers 181, 964-973. DOI: 10.1016/j.carbpol.2017.11.053

Chen, J., Niu, X., Dai, T., Hua, H., Feng, S., Liu, C., McClements, D. J., and Liang, R. (2020). "Amino acid-amidated pectin: Preparation and characterization," Food Chemistry 309, 125768. DOI: 10.1016/j.foodchem.2019.125768

Diaz, J. V., Anthon, G. E., and Barrett, D. M. (2007) "Nonenzymatic degradation of citrus pectin and pectate during prolonged heating: Effects of $\mathrm{pH}$, temperature, and degree of methyl esterification," Journal of Agricultural and Food Chemistry 55(13), 5131-5136. DOI: 10.1021/jf0701483

Einhorn-Stoll, U., Salazar, T., Jaafar, B., and Kunzek, H. (2001). "Thermodynamic compatibility of sodium caseinate with different pectins. Influence of the milieu conditions and pectin modifications," Nahrung/Food 45(5), 332. DOI: 10.1002/1521-3803(20011001)45:5<332::AID-FOOD332>3.0.CO;2-G

Eliyahu, S., Galitsky, A., Ritov, E., and Bianco-Peled, H. (2021). "Hybrid acrylated chitosan and thiolated pectin cross-linked hydrogels with tunable properties," Polymers 13(2), 266. DOI: 10.3390/polym13020266 
EPST (2011). "Degree of substitution," Encyclopedia of Polymer Science and Technology 4, 697-698. DOI: 10.1002/0471440264.pst445

Fan, L., Gao, S., Wang, L., Wu, P., Cao, M., Zheng, H., Xie, W., and Zhou, J. (2011). "Synthesis and anticoagulant activity of pectin sulfates," Journal of Applied Polymer Science 124(3), 2171-2178. DOI: 10.1002/app.35239

Fan, L., Cao, M., Gao, S., Wang, W., Peng, K., Tan, C., Wen, F., Tao, S., and Xie, W. (2012). "Preparation and characterization of a quaternary ammonium derivative of pectin," Carbohydrate Polymers 88(2), 707-712. DOI: 10.1016/j.carbpol.2012.01.021

Flutto, L. (2003) "Pectin: Properties and determination," Encyclopedia of Food Sciences and Nutrition (Second Edition), Academic Press, 4440-4449. DOI: 10.1016/B0-12-227055-X/00901-9

Fraenkel-Conrat, H., and Olcott, H. S. (1944). "Esterification of fatty and amino acids with 1,2-epoxides in aqueous solution," Journal of the American Chemical Society 66(8), 1420-1421. DOI: 10.1021/ja01236a503

Fu, J., and in het Panhuis, M. (2019). "Hydrogel properties and applications," Journal of Materials Chemistry B 7(10), 1523-1525. DOI: 10.1039/C9TB90023C

Fukaya, Y., Sugimoto, A., and Ohno, H. (2006). "Superior solubility of polysaccharides in low viscosity, polar, and halogen-free 1,3-dialkylimidazolium formates," Biomacromolecules 7(12), 3295-3297. DOI: 10.1021/bm060327d

Gao, W., Lin, X., Lin, X., Ding, J., Huang, X., and Wu, H. (2011). "Preparation of nano-sized flake carboxymethyl cassava starch under ultrasonic irradiation," Carbohydrate Polymers 84, 1413-1418. DOI: 10.1016/j.carbpol.2011.01.056

Guo, J., Zhang, J., Yue, Y., and Guo, Y. (2014). "Mechanism of mercury removal by a novel hydrazine hydrate-modified pectin," Bulgarian Chemical Communications 46(4), 801-805.

Gupta, B., Tummalapalli, M., Deopura, B., and Alam, M. S. (2013). "Functionalization of pectin by periodate oxidation," Carbohydrate Polymers 98(1), 1160-1165. DOI: 10.1016/j.carbpol.2013.06.069

Hauptstein, S., Hintzen, F., Müller, C., Ohm, M., and Bernkop-Schnürch, A. (2013). "Development and in vitro evaluation of a buccal drug delivery system based on preactivated thiolated pectin," Drug Development and Industrial Pharmacy 40(11), 1530-1537. DOI: 10.3109/03639045.2013.836213

Heinze, T., ElSeoud, O. A., and Koschella, A. (2018). Cellulose Derivatives: Synthesis, Structure, and Properties, Springer International Publishing, XXIX-531. DOI: $10.1007 / 978-3-319-73168-1$

Hennink, W. E., and van Nostrum, C. F. (2012). "Novel crosslinking methods to design hydrogels," Advanced Drug Delivery Reviews 64, 223-236. DOI: 10.1016/j.addr.2012.09.009

Hintzen, F., Hauptstein, S., Perera, G., and Bernkop-Schnürch, A. (2013). "Synthesis and in vitro characterization of entirely S-protected thiolated pectin for drug delivery," European Journal of Pharmaceutics and Biopharmaceutics 85(3), 12661273. DOI: $10.1016 / j . e j p b .2013 .09 .017$

Hou, W.-C., Lee, M.-H., Hsu, F.-L., and Lin, Y.-H. (2003). "Inhibitory activities of semicarbazide-sensitive amine oxidase and angiotensin converting enzyme of pectin hydroxamic acid," Journal of Agricultural and Food Chemistry 51, 63626366. DOI: 10.1021/jf034463a

Karaki, N., Aljawish, A., Muniglia, L., Bouguet-Bonnet, S., Leclerc, S., Paris, C., Jasniewski, J., and Humeau-Virot, C. (2017). "Functionalization of pectin with laccase-mediated oxidation products of ferulic acid," Enzyme and Microbial Technology 104, 1-8. DOI: 10.1016/j.enzmictec.2017.05.001 
Khan, Z., Minas, M. U., Ahmad. M., Khan K. U., Sohail, M., and Khalid, I. (2019). "Functionalized pectin hydrogels by cross-linking with monomer: Synthesis, characterization, drug release and pectinase degradation studies," Polymer Bulletin 77(1), 339-356. DOI: 10.1007/s00289-019-02745-8

Kothandaraman, G. P., Ravichandran, V., Bories, C., Loiseau, P. M., and Jayakrishnan, A. (2017). "Anti-fungal and anti-leishmanial activities of pectinamphotericin B conjugates," Journal of Drug Delivery Science and Technology 39, 1-7. DOI: 10.1016/j.jddst.2017.02.010

Kouwijzer, M., Schols, H., and Pérez, S. (1996). "Acetylation of rhamnogalacturonan I and homogalacturonan: Theoretical calculations," Progress in Biotechnology 14, 57-65. DOI: 10.1016/S0921-0423(96)80246-2

Krall, S. M., and McFeeters, R. F. (1998). "Pectin hydrolysis: Effect of temperature, degree of methylation, $\mathrm{pH}$, and calcium on hydrolysis rates," Journal of Agricultural and Food Chemistry 46(4), 1311-1315. DOI: 10.1021/jf970473y

Kurita, O., Miyake, Y., and Yamazaki, E. (2012). "Chemical modification of citrus pectin to improve its dissolution into water," Carbohydrate Polymers 87(2), 17201727. DOI: 10.1016/j.carbpol.2011.09.081

Liang, R.-H., Wang, L.-H., Chen, J., Liu, W., and Liu, C. M. (2015). "Alkylated pectin: Synthesis, characterization, viscosity and emulsifying properties," Food Hydrocolloides 50, 65-73. DOI: 10.1016/j.foodhyd.2015.04.007

Lin, C.-Y., Peng, H.-H., Chen, M.-H., Sun, J.-S., Liu, T.-Y., and Chen, M.-H. (2015). "In situ forming hydrogel composed of hyaluronate and polygalacturonic acid for prevention of peridural fibrosis," Journal of Materials Science: Materials in Medicine 26(4), 168-180. DOI: 10.1007/s10856-015-5478-3

Lin, C.-Y., Peng, H.-H., Chen, M.-H., Sun, J.-S., Liu, T.-Y., and Chen, M.-H. (2016). "Ibuprofen-conjugated hyaluronate/polygalacturonic acid hydrogel for the prevention of epidural fibrosis," Journal of Biomaterials Applications 30(10), 1589-1600. DOI: 10.1177/0885328216635838

Lin, J., Yu, S., Ai, C., Zhang, T., and Guo, X. (2020). "Emulsion stability of sugar beet pectin increased by genipin crosslinking," Food Hydrocolloids 101, 105459. DOI: 10.1016/j.foodhyd.2019.105459

Manickam, B., Sreedharan, R., and Elumalai, M. (2014). “Genipin' - The natural water soluble cross-linking agent and its importance in the modified drug delivery systems: An overview," Current Drug Delivery 11(1), 139-145. DOI: $10.2174 / 15672018113106660059$

Mohen, D. (2008). "Pectin structure and biosynthesis," Current Opinion in Plant Biology 11(3), 266-277. DOI: 10.1016/j.pbi.2008.03.006

Monfregola, L., Leone, M., Vittoria, V., Amodeo, P., and Luca, S. D. (2011). "Chemical modification of pectin: environmental friendly process for new potential material development," Polymer Chemistry 2, 800-804. DOI: 10.1039/c0py00341g

Morris, G. A., and Binhamad, H. A. S. (2020). "Isolation and characterisation of pectin," in: Pectin: Technological and Physiological Properties, V. Kontogiorgos (ed.), Springer International Publishing, 61-82. DOI 10.1007/978-3-030-53421-9_4

Mortensen, A., Aguilar, F., Crebelli, R., Di Domenico, A., Dusemund, B., Frutos, M. J., Galtier, P., Gott, D., Gundert-Remy, U., Lambré, C., Leblanc, J.-C., Lindtner, O., Moldeus, P., Mosesso, P., Oskarsson, A., Parent-Massin, D., Stankovic, I., Waalkens-Berendsen, I., Wright, M., Younes, M., Tobback, P., Ioannidou, S., Tasiopoulou, S., and Woutersen, R. A. (2017). "Re-evaluation of pectin (E440i) and amidated pectin (E440ii) as food additives" European Food Safety Authority Journal 15, e04866. DOI: 10.2903/j.efsa.2017.4866 
Moss, G. P., Smith, P. A. S., and Tavernier, D. (1995). "Glossary of class names of organic compounds and reactivity intermediates based on structure (IUPAC Recommendations 1995)," Pure and Applied Chemistry 67(8-9), 1307-1375. DOI: $10.1351 /$ pac199567081307

Muller, P. (1994). "Glossary of terms used in physical organic chemistry (IUPAC Recommendations 1994)." Pure and Applied Chemistry 66(5), 1077-1184. DOI: 10.1351/pac199466051077

Müller-Maatsch, J., Caligiani, A., Tedeschi, T., Elst, K., and Sforza, S. (2014). "Simple and validated quantitative $1 \mathrm{H}$ NMR method for the determination of methylation, acetylation, and feruloylation degree of pectin," Journal of Agricultural and Food Chemistry 62(37), 9081-9087. DOI: 10.1021/jf502679s

Muthukumaran, C., Kanmani, B. R., Sharmila, G., Manoj Kumar, N., and Shanmugaprakash, M. (2018). "Carboxymethylation of pectin: Optimization, characterization and in-vitro drug release studies," Carbohydrate Polymers 194, 311-318. DOI: 10.1016/j.carbpol.2018.04.042

Peng, H.-H., Chen, J.-W., Yang, T.-P., Kuo, C.-F., Wang, Y.-J., and Lee, M.-W. (2011). "Polygalacturonic acid hydrogel with short-chain hyaluronate cross-linker to prevent postoperative adhesion," Journal of Bioactive and Compatible Polymers 26(6), 552-564. DOI: 10.1177/0883911511423562

Peng, H.-H., Chen, Y.-M., Lee, C.-I., and Lee, M.-W. (2013). "Synthesis of a disulfide cross-linked polygalacturonic acid hydrogel for biomedical applications," Journal of Materials Science: Materials in Medicine 24 (6), 1375-1382. DOI: 10.1007/s10856-013-4901-x

Perera, G., Hombach, J., and Bernkop-Schnürch, A. (2010). "Hydrophobic thiolation of pectin with 4-aminothiophenol: Synthesis and in vitro characterization," AAPS Pharmaceutical Science and Technology 11(1), 174-180. DOI: 10.1208/s12249009-9370-7

Perumal, P., Selvin, P. C., and Selvasekarapandian, S. (2018). "Characterization of biopolymer pectin with lithium chloride and its applications to electrochemical devices," Ionics 24(10), 3259-3270. DOI: 10.1007/s11581-018-2507-5

Plewa, A., Niemiec, W., Filipowska, J., Osyczka, A. M., Lach, R., Szczubiałka, K., and Nowakowska, M. (2011). "Photocrosslinkable diazoresin/pectin films Synthesis and application as cell culture supports," European Polymer Journal 47, 1503-1513. DOI: 10.1016/j.eurpolymj.2011.06.002

Renard, C. M. G. C., and Jarvis, M. C. (1999). "Acetylation and methylation of homogalacturonans. 1: Optimization of the reaction and characterization of the products," Carbohydrate Polymers 39(3), 201-207. DOI: 10.1016/S01448617(99)00006-5

Ropartz, D., and Ralet, M.-C. (2020). "Pectin structure," Pectin: Technological and Physiological Properties (Springer International Publishing), 17-36. DOI: 10.1007/978-3-030-53421-9_2

Saito, G., Swanson, J. A., and Lee, K.-D. (2003). Drug delivery strategy utilizing conjugation via reversible disulfide linkages: Role and site of cellular reducing activities," Advanced Drug Delivery Reviews 55(2), 199-215. DOI: 10.1016/S0169-409X(02)00179-5

Schöniger, W. (1956). "Die mikroanalytische Schnellbestimmung von Halogenen und Schwefel in organischen Verbindungen," Microchimica. Acta 44, 869-876. DOI: 10.1007/BF01262130

Seslija, S., Spasojević, P., Panić, V., Dobrzy’nska-Mizera, M., Immirzi, B., Stevanović, J., and Popović, I. (2018). "Physico-chemical evaluation of hydrophobically modified pectin derivatives: Step toward application," 
International Journal of Biological Macromolecules 113, 924-932. DOI: 10.1016/j.ijbiomac.2018.03.006

Seymour, C., and Knox, P. (2002). Pectins and Their Manipulation, Blackwell Publishing, Oxford. ISBN 1-841-27228-0

Sharma, R., and Ahuja, M. (2011). "Thiolated pectin: Synthesis, characterization and evaluation as a mucoadhesive polymer," Carbohydrate Polymers 85(3), 658-663. DOI: 10.1016/j.carbpol.2011.03.034

Shi, X., Cui, S., Song, X., Rickel, A. P., Sanyour, H. J., Zheng, J., Hu, J., Hong, Z., Zhou, Y., and Liu, Y. (2020). "Gelatin-crosslinked pectin nanofiber mats allowing cell infiltration," Materials Science and Engineering: C 112, 110941. DOI: 10.1016/j.msec.2020.110941

Smitha, B., Sridhar, S., and Khan, A. A. (2005). "Chitosan-sodium alginate polyion complexes as fuel cell membranes," European Polymer Journal 41(8), 1859-1866. DOI: 10.1016/j.eurpolymj.2005.02.018

Souto-Maior, J. F. A., Reis, A. V., Pedreiro, L. N., and Cavalcanti, O. A. (2009). "Phosphated crosslinked pectin as a potential excipient for specific drug delivery: preparation and physicochemical characterization," Polymer International 59(1), 127-135. DOI: 10.1002/pi.2700

Tang, X.-H., Xie, P., Ding, Y., Chu, L.-Y., Hou, J.-P., Yang, J.-L., Song, X., and Xie, Y.-M. (2010). "Synthesis, characterization, and in vitro and in vivo evaluation of a novel pectin-adriamycin conjugate," Bioorganic and Medicinal Chemistry 18(4), 1599-1609. DOI: 10.1016/j.bmc.2009.12.076

Vahini, M., Muthuvinayagam, M., and Isa, M. I. N. (2019). "Preparation and characterization of biopolymer electrolytes based on pectin and $\mathrm{NaNO}_{3}$ for battery applications," Polymer Science, Series A 61(6), 823-831. DOI: 10.1134/S0965545X19060129

Vityazev, F. V., Golovchenko, V. V., Patova, O. A., Drozd, N. N., Makarov, V. A., Shashkov, A. S., and Ovodov, Y. S. (2010). "Synthesis of sulfated pectins and their anticoagulant activity," Biochemistry Moscow 75(6), 759-768. DOI: 10.1134/S0006297910060118

Wang, R., Liang, R., Dai, T., Chen, J., Shuai, X., and Liu, C. (2019). "Pectin-based adsorbents for heavy metal ions: A review," Trends in Food Science \& Technology 91, 319-329. DOI: 10.1016/j.tifs.2019.07.033

Wang, J.-T., Wan, C.-C., and Hong, J.-L. (2020). "Polymer blends of pectin/poly(acrylic acid) as efficient binders for silicon anodes in lithium-ion batteries," ChemElectroChem 7(14), 3106-3115. DOI: 10.1002/celc.202000666

Williams, M. A. K. (2020). "Pectin gelation and its assembly into functional materials," in: Pectin: Technological and Physiological Properties, Springer International Publishing, pp. 125-148. DOI: 10.1007/978-3-030-53421-9_7

Würfel, H., Kayser, M., and Heinze, T. (2019a). "Trimethylsilylation of polygalacturonic acid," Macromolecular Chemistry and Physics 220(9), article no. 1900002. DOI: 10.1002/macp.201900002

Würfel, H., Kayser, M., and Heinze, T. (2019b). "Non-aqueous solvent for efficient dissolution of polygalacturonic acid," Carbohydrate Polymers 207, 791-795. DOI: 10.1016/j.carbpol.2018.12.036

Würfel, H., Geitel, K., and Heinze, T. (2021). "Efficient heterogeneous synthesis of reactive polygalacturonic acid hydrazides," Carbohydrate Polymers 261, article no. 117838. DOI: 10.1016/j.carbpol.2021.117838

Zhang, G., Zheng, C., Huang, B., and Fei, P. (2020a). "Preparation of acylated pectin with gallic acid through enzymatic method and their emulsifying properties, 
antioxidation activities and antibacterial activities," International Journal of Biological Macromolecules 165, 198-204. DOI: 10.1016/j.ijbiomac.2020.09.195

Zhang, G., Huang, B., Zheng, C., Chen, Q., and Fei, P. (2020b). "Investigation of a lipase-catalyzed reaction between pectin and salicylic acid and its isomers and evaluation of the emulsifying properties, antioxidant activities, and antibacterial activities of the corresponding products," Journal of Agricultural and Food Chemistry 69, 1234-1241. DOI: 10.1021/acs.jafc.0c06120

Zheng, X. F., Lian, Q., Yang, H., Jia, D. D., and Wang, D. J. (2013). "Syntheses and characterization of alkyl-pectin materials," Asian Journal of Chemistry 25, 21212124. DOI: 10.14233/ajchem.2013.13353

Article submitted: June 28, 2021; Peer review completed: August 2, 2021; Revised version received and accepted: August 16, 2021; Published: August 30, 2021.

DOI: 10.15376/biores.16.4.Wurfel 